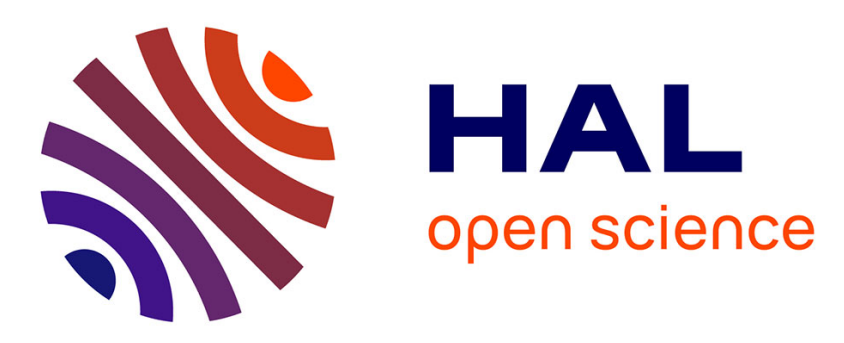

\title{
Nonlinear dynamics of uniformly loaded Elastica: Experimental and numerical evidence of motion around curled stable equilibrium configurations
}

\author{
Djebar Baroudi, Ivan Giorgio, Antonio Battista, Emilio Turco, Leonid A
} Igumnov

\section{To cite this version:}

Djebar Baroudi, Ivan Giorgio, Antonio Battista, Emilio Turco, Leonid A Igumnov. Nonlinear dynamics of uniformly loaded Elastica: Experimental and numerical evidence of motion around curled stable equilibrium configurations. Journal of Applied Mathematics and Mechanics / Zeitschrift für Angewandte Mathematik und Mechanik, In press, 10.1002/zamm.201800121 . hal-02108277

\section{HAL Id: hal-02108277 \\ https://hal.science/hal-02108277}

Submitted on 13 May 2019

HAL is a multi-disciplinary open access archive for the deposit and dissemination of scientific research documents, whether they are published or not. The documents may come from teaching and research institutions in France or abroad, or from public or private research centers.
L'archive ouverte pluridisciplinaire HAL, est destinée au dépôt et à la diffusion de documents scientifiques de niveau recherche, publiés ou non, émanant des établissements d'enseignement et de recherche français ou étrangers, des laboratoires publics ou privés. 


\title{
Nonlinear dynamics of uniformly loaded Elastica: Experimental and numerical evidence of motion around curled stable equilibrium config- urations
}

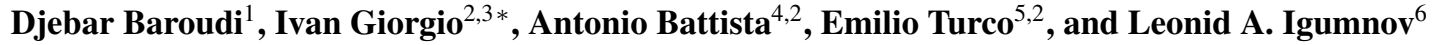 \\ ${ }^{1}$ Aalto University School of Engineering, Aalto University, FIN-00076 Aalto, Finland. \\ 2 International Research Center for the Mathematics and Mechanics of Complex Systems, Università degli studi \\ dell'Aquila, 67100 L'Aquila, Italy. \\ ${ }^{3}$ Department of Structural and Geotechnical Engineering, SAPIENZA Università di Roma, 00184 Rome, Italy. \\ ${ }^{4}$ Université de La Rochelle, La Rochelle, France. \\ ${ }^{5}$ Department of Architecture, Design and Urban planning, University of Sassari, 07041 Alghero, Italy. \\ ${ }^{6}$ Research Institute for Mechanics, Lobachevsky State University of Nizhni Novgorod, Nizhni Novgorod, Russian Feder- \\ ation.
}

Received XXXX, revised XXXX, accepted XXXX

Published online XXXX

Key words Nonlinear beam, Hencky bar-chain, discrete modelling

MSC (2010) $00-\mathrm{xx}$

\author{
"Frustra fit per plura quod fieri potest per pauciora." \\ (It is pointless to do with more what can be done with fewer.) \\ In Summa Totius Logicae by William of Ockham
}

\begin{abstract}
It has been numerically observed and mathematically proven that for a clamped Euler's Elastica, which is uniformly loaded, there exist, in large deformations, some 'undocumented' equilibrium configurations which resemble a curled pending wire. Even if Elastica is one of the most studied model in mathematical physics, we could not find in the literature any description of an equilibrium like the one whose existence was forecast theoretically in [36].

In this paper, we prove that this kind of equilibrium configurations can be actually observed experimentally when using 'soft' beams. We mean with soft beams: Elasticae whose ratio between the applied load intensity and the bending stiffness is large enough. Moreover, we prove experimentally that such equilibrium configurations are actually stable, by observing their oscillations around the considered nonstandard equilibrium configuration.

To describe theoretically such oscillations we consider, instead of a 'soft' Elastica model, directly a Hencky-type discrete model, i.e. a 'masses-springs' finite dimensional Lagrangian model. In this way we formulate, avoiding the use of an intermediate continuum model, a model for which numerical simulations can be performed without the introduction of any further discretization. In this way, we can also predict quantitatively the motions of soft beams, in the regime of large displacements and deformations. Postponing to future investigations more careful quantitative measurements, we report here that it was possible to get a rather promising qualitative agreement between observed motions and predictive numerical simulations.
\end{abstract}

\section{Introduction}

The fundamental works by Bernoulli and Euler [25,63], introduced the concept of Elastica in mathematical physics. This model has been the basis of many mathematical investigations and leads to important technological applications. It constitutes from the mathematical point an interesting source of theoretical and experimental problems [27] while it had applications to structural mechanics which had a tremendous impact. The literature in which the theory of Euler-Bernoulli beam theory and/or its applications are studied is so huge that it is probably impossible to give a reasonable account for it. Among the many relevant problems which have been studied with great details and care we, simply as they are instrumental to our investigations, cite $[8,13,62,71,94,102,105]$.

\footnotetext{
* Corresponding author, E-mail: ivan.giorgio@uniroma1.it
} 
However, the importance of the model is so great that there are still left interesting and practically significant problems to be studied anew or whose study needs to be completed. In particular, the problem of large deformations of an Euler beam under loads distributed along its length seems not to have attracted the attention which it deserved. This problem played a relevant role in the theory of metamaterials and in particular when dealing with pantographic microstructures with long beams $[12,29,34,35,40,48,51,61,104]$. In pantographic sheets $[6,43,69]$ the single fiber, in a certain class of situations, can be modelled as a beam interacting with the other beams/fibers, in such a way that in the homogenized limit, a distributed load can be assumed to be applied. Actually in pantographic structures with long 'fibres' (see, e.g., Fig. 1), we can observe the existence of 'long and soft beams' undergoing large deformations and subjected to 'distributed' loads. Of course, such beams are not always exactly subjected to uniformly distributed load! However, it was believed that a better knowledge of the possible equilibrium shapes for these load cases was necessary to proceed in the investigations about pantographic metamaterials. It has to be remarked that, when studying large deformations of uniformly loaded Euler beams one is led to non-autonomous variational problems, i.e., to variational problems whose corresponding Euler-Lagrange equations are non-autonomous differential equations. These problems cannot be solved easily, standing the available mathematical techniques: in fact in [36] it could be shown that: i) there exist non-trivial equilibrium shapes having a curled shape for clamped Euler beams under uniformly distributed dead load orthogonal to the initial straight undeformed configuration ii) many interesting properties of the global and local minimizers of the total energy for Euler's elastica hold, in the case of large displacements and deformations equilibria.

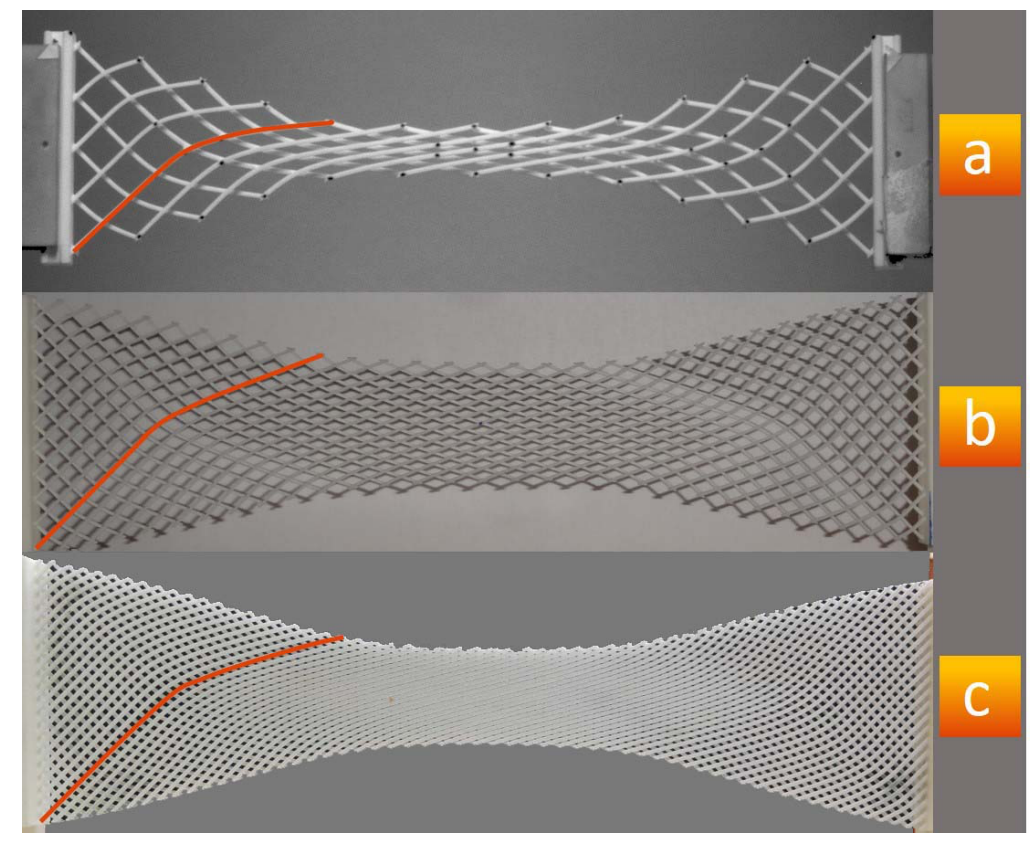

Fig. 1 Some illustrative examples of pantographic structures under a bias test varying the density of the fibers.

Recently a reassessment of the models needed to describe beam-like structural elements has been used in order to simplify the tools needed to study nonlinear equilibrium shapes, loss of stability, buckling and post-buckling phenomena like those studied in $[64,83,84,95]$. In particular in order to simplify the computation of the buckling load of a beam, its large deformations and its multiple stable equilibria discrete Hencky models have been recovered [16, 17, 111, 114]. Indeed Hencky, in his seminal work [72], for making possible some semi-analytical solutions of the problem of finding large deformations of Euler beams, proposed a very clever and effective way leading to the computation of the buckling load of a rectilinear planar beam. ${ }^{1}$

The idea, in a nutshell, is based on the choice of modeling a beam as a system of rigid linking elements interconnected by elastic joints. Using Lagrangian generalized coordinates and Lagrangian potential energy for the elastic joints by minimizing total energy for the so obtained system one manages to find a finite set of equilibrium equations, i.e. the conditions imposing that the gradient of the total potential energy is vanishing. In this way, one gets rather simply an effective estimate of the buckling load. Obviously, such an estimate has an increasing accuracy when one increases the number of elastic joints and rigid links.

\footnotetext{
1 For completeness we recall here that Hencky's idea is already present in the works of Gabrio Piola almost one century before, see [37,46], together with the main ideas needed to get via homogenization, from discrete Lagrangian systems, generalized, and eventually non-local, continua (see [15, 38]).
} 
From a conceptual point of view, possibly the most attractive feature of Hencky's procedure consists in the fact that it avoids at all the necessity of introducing continuum models. As continuous models, to get numerical results in large deformations, need, at the end, to be discretized one gets a relevant economy in the modeling procedure. Indeed, the Hencky model is discrete since the very beginning and it has a clear mechanical interpretation. When applied to systems which need generalized continua, to use discrete models may also avoid some sharp debates $[39,41,45,47,49,50]$.

To make this discussion complete, and make happy those who consider continuum models more important, we can recall here that recently some $\Gamma$-convergence results have been proven that Hencky's model is a fully reliable approximation of continuous inextensible [5] and extensible [4] Euler-Bernoulli beams. Actually generalized continua can be proven to be $\Gamma$-limits of systems described at micro-level as first gradient continua [2, 3, 91,92]. On the other hand, it is possible to regard a beam as an energetic boundary curve on a two-dimensional manifold in a three-dimensional space and employ the frameworks developed in [73,77,78] and even account for higher gradients elaborated in [75]. The advantage of this approach is that the fictitious bulk material can regularize the behavior of the beam and this allows to analyze buckling in a computational framework. Furthermore, the substrate provides a versatile medium to assign distributed loads on the beam similar to thin films on elastic foundations [74, 76, 89].

A further remark is needed here: the introduced discrete model is intrinsically nonlinear. It naturally incorporates geometrical and material non-linearities: this is the reason for which, also when one linearizes it, he can avoid all the issues implied by the loss of objectivity of the energy when, in the neighborhood of a certain equilibrium configuration, the linearization is performed.

Limiting our attention to mechanical problem and choosing, in the literature those works whose spirit is closer to ours, the application of Hencky's modeling concept can be found again in some more recent works which deal with single beams, see $[10,11,26,32,58,81,99,100,106]$, or with assemblages of beams, see [44, 48, 59, 69, 107, 112]. Moreover Hencky-type models can be used to conceive specially targeted lattice materials structured at different scales, see [22]. An additional reason suggesting to consider Hencky's discrete models is given exactly by the consideration of the aforementioned architectured multiscale materials (whose microstructure is often modeled as an assembly of beams, [28, 54, 55, 101]). Indeed, this kind of micro-structured materials are constituted by a very large number of structural elements, and therefore some reduced order masses-springs models can be useful for their description.

It has to be also remarked that when one studies complex metamaterials [21, 29, 39, 44, 48, 53, 86, 87], and if one wants to use generalized continuum models finally he is led to the problem of synthesis: i.e. the problem of determining a microstructure governed, in a suitable homogenization limit, by postulated equations. This synthesis is finally obtained by means of lattices structures, which can be regarded as a kind of generalization of the discrete systems introduced by Hencky. As these discrete models have been proven to be really efficient in predicting the mechanical behavior of considered structures, (see, e.g., [57] where different shapes of the unit cells are considered, [79, 80] for triangular lattices and $[56,96]$ for chiral lattices) one can really question the need of talking at all about continuum models. We can, therefore, deduce that the understanding of the pattern of deformations of Euler beams, which are the fundamental structural elements considered in meta-material micro-structures, is a very important topic in the theory of metamaterials, may one decide to model them as e.g. Elasticae or as Hencky-type discrete systems.

These general considerations apply also to the more particular class of pantographic (micro)structures [44, 109], particularly useful when looking for materials showing an elastic behavior in the range of large displacements and large deformations, see [107, 110]. It has to be remarked that the effectiveness of Hencky-type models allows for the inclusion, in the modelling scheme, also of the models for the onset of any form of damage phenomenon eventually leading to failure phenomena $[103,108]$.

In the literature, one can find some examples of works dealing with the nonlinear dynamics of beams, modelled as discrete models (see for instance [59,68,81,112]). Therefore, the present work is aimed to contribute to an effort which has been already initiated. One of the main novelty consists in having adapted the approach already presented in the literature to the aim of describing very simple (but probably not already considered) physical experiments, which can be reproduced without expensive tools: we mean the construction of soft (highly flexible) beams, their placing in a curled equilibrium shape under a gravity load and the investigation of their planar nonlinear motion in the neighborhood of this 'nonstandard' configuration.

We assume, in formulating our Hencky-type model, the same hypotheses which are peculiar of the continuous Euler beam model: i) the conservative part of deformation energy (i.e. elastic energy) depends upon the relative Hencky-bars rotations (i.e. curvature); ii) the Hencky bars are rigid (i.e. the axis of the Euler beam is inextensible); iii) because of the simplicity of the Hencky model then the concept of shear deformation cannot even be formulated when considering the discrete model, this situation corresponds to the assumption that the the cross-section with respect to the axis of the used soft beams is negligible; and that this cross-section must be regarded to be not deformable.

As we will see with numerical experiments, Hencky's model is consistent with the assumptions usually accepted for formulating Euler beam model. 
For determining the equilibrium shapes of soft beams, and the numerical simulations we can best fit mechanical parameters necessary to specify the particular model for the specific soft beam used in the experimental setup. Using these parameters, i.e. the masses and the stiffnesses of rotational elastic joints, and fitting subsequently only the damping coefficients we can describe, by running efficient and robust numerical codes, rather effectively the observed mechanical behavior and can highlight the main and peculiar features of the dynamic nonlinear behavior of the considered class of soft beams.

The plan of the paper is the following: after this short introduction, the main technical ideas which necessary to construct the used discrete Hencky model are described in Sec. 2. In the subsequent Sec. 3, we carefully describe the experimental set-up which we have used and the two specific experiments which were performed. The obtained data are then fully reported, making thus possible the detailed comparison between experimental evidence and the predictions given by the numerical simulations which were performed, both for static equilibrium configurations and for the observed motions. Finally, in Section 4 the paper is concluded, by discussing the impact of our main results in the static and dynamics of metamaterials together with the possible extensions of this work to suitably newly designed linear metamaterials.

\section{An Hencky-type model replacing the Elastica model}

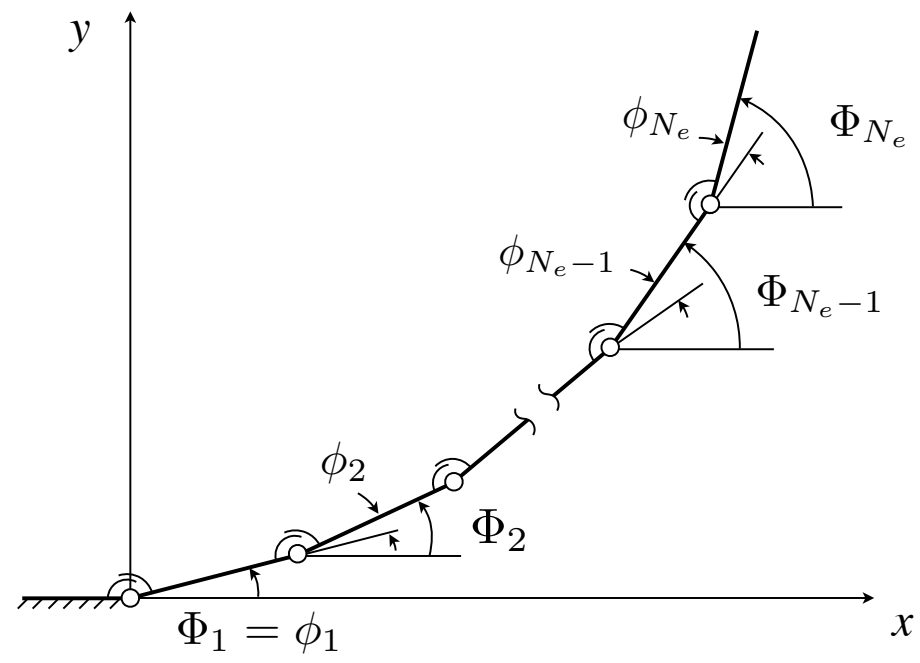

Fig. 2 Hencky-type lumped mass-spring model for a highly flexible beam.

We want to describe the static and dynamic behavior of a 'soft' cantilever beam to which a gravity load is applied, in the most simple possible way, having in mind the need of describing a specific set of experimental pieces of evidence (see next section).

The choice made here, assuming a point of view accepted also by Piola [37, 38,41,46], is based exactly on a similar choice made by Hencky. One could consider Hencky-type discrete model as an independent model, having its own physical meaning and its own numerical advantages, or as a discrete approximation of Euler, e.g., Elastica.

By simply adapting the Hencky technique [98,111,114] we choose to consider a discrete system made of an 'articulated' sequence of $N_{e}$ (rigid, as the beam is assumed inextensible) rods of length $\eta$, which are constrained at their terminal points by perfect hinges, i.e. hinges which are exerting vanishing couples. To each joint we assume that a rotational spring is applied, aiming to model the elasticity effects which are resistant to the bending of the considered mechanical system. (see Fig. 2). Each Lagrangian configuration of the just described system is fully specified once it is known the time evolution of the $N_{e}$ Lagrangian angular coordinates, $\Phi_{i}(t)$. These coordinates determine the orientation of each of the considered rigid rods relative to the $x$-axis which is chosen to be horizontal (the applied loads will be assumed to be vertical). The direction along which the applied loads are assumed to be directed coincides with the $y$-axis, which is oriented vertically upwards.

The set of bars, before the deformation, is assumed to be straight along the $x$-axis. The springs will have their rest length such that this configuration will have the minimum of deformation energy.

To each rigid segment, it is attributed a mass, $m_{i}$, together with a moment of inertia, $J_{i}$, relatively to a rotation axis which is orthogonal to the plane in which the motion is assumed to be constrained. This rotation axis is assumed to pass through the center of mass of the bar. 
The position of the mass center for each segment is given by the following formula:

$$
\left\{\begin{array}{l}
x_{i}(t)=\sum_{k=1}^{i} \eta\left(1-\frac{\delta_{i k}}{2}\right) \cos \left(\Phi_{k}(t)\right) \\
y_{i}(t)=\sum_{k=1}^{i} \eta\left(1-\frac{\delta_{i k}}{2}\right) \sin \left(\Phi_{k}(t)\right)
\end{array}\right.
$$

where $\delta_{i k}$ is the Kronecker delta.

In order to evaluate the velocities of said mass centers, one must differentiate with respect to time, getting the following formulae:

$$
\left\{\begin{array}{l}
\dot{x}_{i}(t)=-\sum_{k=1}^{i} \eta\left(1-\frac{\delta_{i k}}{2}\right) \dot{\Phi}_{k}(t) \sin \left(\Phi_{k}(t)\right) \\
\dot{y}_{i}(t)=\sum_{k=1}^{i} \eta\left(1-\frac{\delta_{i k}}{2}\right) \dot{\Phi}_{k}(t) \cos \left(\Phi_{k}(t)\right)
\end{array}\right.
$$

The adopted Lagrangian parameters are suitable to produce a really convenient expression for the equations of motions which we will derive starting from the Action Principle applied using as a starting point the following Lagrangian, which, also because of the analysis by Hamilton, and specifically by Piola (see [37]) for Euler beams, we believe is suitable in the considered physical instance

$$
\mathscr{L}=\mathfrak{K}-\Psi
$$

where $\mathfrak{K}$ and $\Psi$ are the kinetic and potential energies of the system, respectively, as determined by the theory of constrained rigid body motions with elastic joints (see, e.g., [14,70]).

It is easy to accept that the potential energy $\Psi$ can be obtained by two terms: an elastic $\Psi_{e l}$ and a gravitational $\Psi_{w g}$ term. The former elastic term $\Psi_{e l}$ is postulated as follows

$$
\Psi_{e l}=\sum_{i=1}^{N_{e}} \kappa_{b i}\left[\cosh \left(\phi_{i}\right)-1\right]
$$

where the relative angles between adjacent rods, $\phi_{i}$, were introduced. Because of the clamping constraint we have: $\phi_{1}=\Phi_{1}$ while $\phi_{i}=\Phi_{i}-\Phi_{i-1}$ for $i \geq 2$. Moreover, we have assumed that the concentrated bending stiffnesses $\kappa_{b i}$, associated to the rotational springs $[43,107]$ are all equal (uniformity of bending stiffness condition) and that the applied external loads reduce to the gravitational term $\Psi_{w g}$ given by

$$
\Psi_{w g}=\sum_{i=1}^{N_{e}} g m_{i}\left[\sum_{k=1}^{i} \eta\left(1-\frac{\delta_{i k}}{2}\right) \sin \left(\Phi_{k}\right)\right]
$$

where $g$ is the acceleration of gravity.

In order to be self-contained, we recall the the kinetic energy, using König's theorem, can be proven to have the following algebraic expression

$$
\mathfrak{K}=\sum_{i=1}^{N_{e}} \frac{1}{2} m_{i}\left\{\left[\sum_{k=1}^{i} \eta\left(1-\frac{\delta_{i k}}{2}\right) \sin \left(\Phi_{k}\right) \dot{\Phi}_{k}\right]^{2}+\left[\sum_{k=1}^{i} \eta\left(1-\frac{\delta_{i k}}{2}\right) \cos \left(\Phi_{k}\right) \dot{\Phi}_{k}\right]^{2}\right\}+\frac{1}{2} J_{i} \dot{\Phi}_{i}^{2}
$$

Because of physical plausibility, the only restriction on the potential energy in Eq. (4) requires that it must be positive definite: therefore any convex function which could be necessary can be postulated. This means that we can expect that some specific meta-beams may be designed being characterized by energies which are not quadratic functions of the relative angles. The choice in Eq. (4) has been made for consistency reasons with the corresponding continuum model, but it is remarkable that the first non-vanishing term in its Taylor expansion valid for small relative variations of angles is exactly the standard usual quadratic form.

We will need to take into account also a possible viscous dissipation, as in our experiments the observed oscillations are damped. (we refer e.g. to [9,33, 66, 82] for an interesting discussion of the concepts needed here). This dissipation occurs mainly, during the motion, because of the interaction of the 'soft' beam with the surrounding air. Therefore we postulate, as a first conjecture and therefore neglecting internal dissipations, a Rayleigh dissipation function of the following form:

$$
\mathfrak{R}=\sum_{i=1}^{N_{e}} \frac{1}{2} c_{b i} \dot{\phi}_{i}^{2}
$$


In conclusions The Euler-Lagrange stationarity condition for chosen action produces the following Lagrange equations of motion

$$
\frac{\partial}{\partial t}\left(\frac{\partial \mathfrak{K}}{\partial \dot{\Phi}_{i}}\right)-\frac{\partial \mathfrak{K}}{\partial \Phi_{i}}+\frac{\partial \Psi_{e l}}{\partial \Phi_{i}}+\frac{\partial \Psi_{w g}}{\partial \Phi_{i}}+\frac{\partial \mathfrak{R}}{\partial \dot{\Phi}_{i}}=0 \quad \text { for } i=1 \ldots N_{e}
$$

The Equations (8) do not present any particular difficulty. It is indeed a system of ordinary differential equations whose existence and uniqueness theorems are well established as are easily available efficient and robust numerical codes. Completely differently would be the nature of the problem to be solved if we had decided to describe the kinematics of our system with an infinite dimensional space of configurations. In this case, the Euler-Lagrange equations governing the motion would have been a partial differential equation whose difficulty is much greater.

We solve numerically the found discrete Lagrange equations in the framework of the computing system Wolfram Mathematica. The solver we have chosen is the one called differential-algebraic system of equations (DAEs). To use it we needed to apply a proper transformation to get an equivalent normal form, $F_{j}\left(t, \Phi_{i}, \dot{\Phi}_{i}\right)=0$. This transformation produces a (larger) set of differential equations which is, instead of the second order, of the first order in the time variable.

\section{Experimental set-up, experimental evidence and obtained numerical simulations}

The previously introduced Lagrangian model is used, in the present section to obtain predictions to be tested with two sets of experimental measurements. To be more precise, we have used two different soft beams constituted by two different materials and having different sizes and lengths. These two soft beams have been sized in order to be assured that the nonstandard equilibrium 'curled' configurations were forecast to be really local minima for their total energy. Moreover, we have chosen the properties of the beams in such a way that the dominant damping factor was actually the friction interaction with the air during the imposed oscillations. In this way, it was possible to investigate successfully the dynamic behavior of used soft beams also in the regime of large oscillation around their effectively observed curled stable equilibrium configurations.

The first used soft beam was constituted by a paper strip. Its dimensions were $329 \times 20 \mathrm{~mm}$, and its thickness was about $0.17 \mathrm{~mm}$. Finally, its mass has been evaluated to be $0.62 \pm 0.02 \mathrm{~g}$.

The second used soft beam was constituted by a rather similar strip but made by a thin isotropic sheet of polyethylene terephthalate, i.e. standard PET. Its dimensions were $220 \times 20 \mathrm{~mm}$ and thickness was about $0.15 \mathrm{~mm}$. The mass of the strip was $0.91 \pm 0.02 \mathrm{~g}$. To show up further effects (which can be easily predicted by simply adding a term in the Lagrangian) we have loaded the free end of the considered beams with another 'concentrated' mass. We simply attached a 'metallic paper clip' of mass $m_{p c}=0.41 \pm 0.02 \mathrm{~g}$ and moment of inertia $J_{p c}=(7.5 \pm 0.5) \times 10^{-9} \mathrm{~kg} \mathrm{~m}^{2}$ passing through a barycentric axis and orthogonal to the plane of motion (see Fig. 3). Specifically, to take into account this paper clip, we add to the Lagrangian function, the gravitational potential energy

$$
\Psi_{p c}=g m_{p c} \sum_{k=1}^{N_{e}} \eta \sin \left(\Phi_{k}\right)
$$

and coherently the kinetic energy

$$
\mathfrak{K}_{p c}=\frac{1}{2} m_{p c}\left\{\left[\sum_{k=1}^{N_{e}} \eta \sin \left(\Phi_{k}\right) \dot{\Phi}_{k}\right]^{2}+\left[\sum_{k=1}^{N_{e}} \eta \cos \left(\Phi_{k}\right) \dot{\Phi}_{k}\right]^{2}\right\}+\frac{1}{2} J_{p c} \dot{\Phi}_{N_{e}}^{2} .
$$

To make the visualization of the shapes easier in the figures we have painted on one elastic strip some lines, while we have colored alternatively with rectangles the other strip.

\subsection{Fitting of the elastic parameters by comparison with the experimental measures of the curled static configurations}

The homogenization results available in the literature assure that the Hencky-type model converges to the Euler-Bernoulli continuous model [5]. In our cases, as $N_{e}=30$ and $\eta=11.0 \mathrm{~mm}$ for the paper strip while $\eta=7.3 \mathrm{~mm}$ for the PET strip, the discrete approximation is close enough to the continuous model. Figure 4 shows a clear convergence analysis made by changing the number of rigid discrete elements from 10 to 40 . It is pretty evident that solutions with 30 and 40 elements are very close, thus we chose $N_{e}=30$ to avoid unnecessary computational burden.

On the other hand, the available solutions to the De Saint Venant problem and the results of homogenization by [24] assure that the Euler bending stiffness can be approximated with the Young modulus of the material constituting the beam 

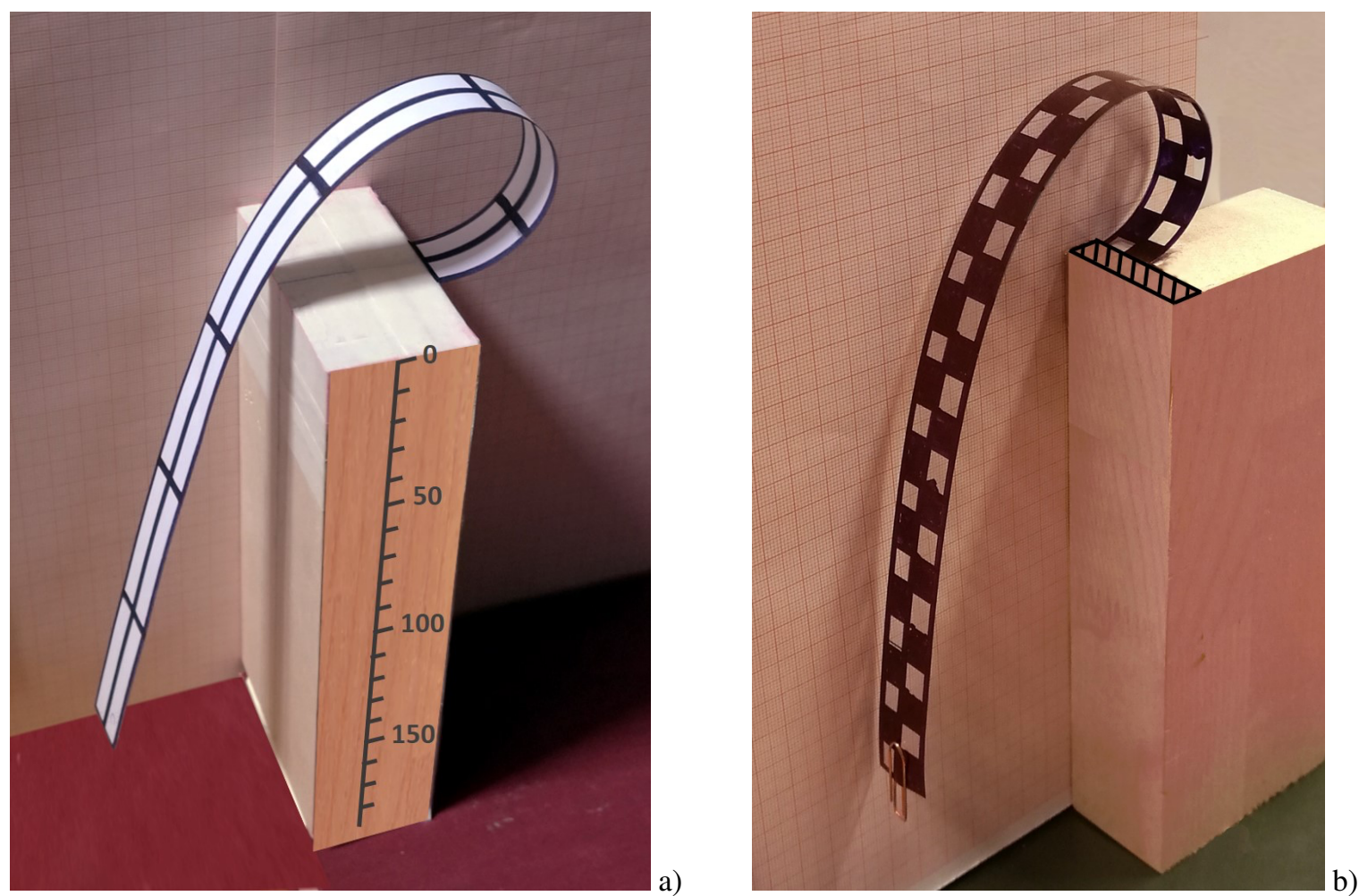

Fig. 3 Stable nonstandard 'curled' equilibrium shapes: paper beam a; PET beam with a mass on the tip b.
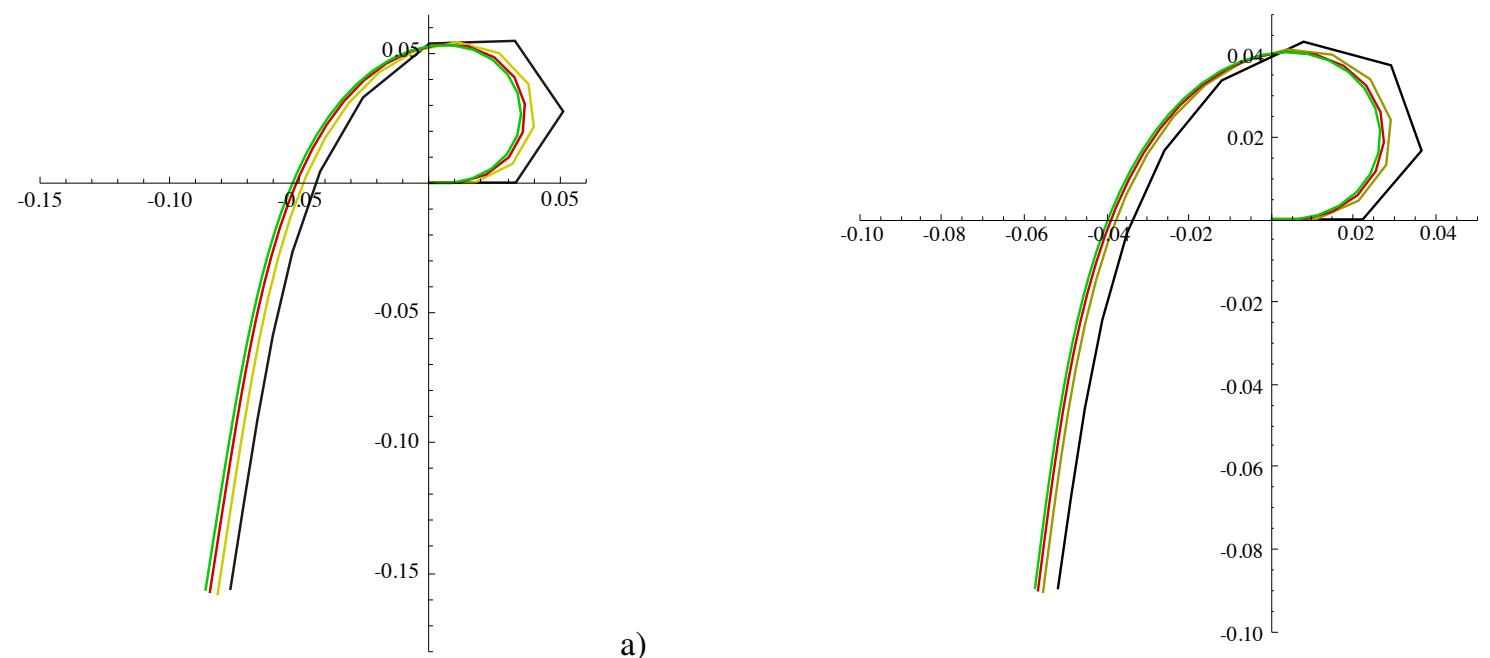

a)

b)

Fig. 4 Convergence analysis of curled stable equilibrium configurations obtained by numerical simulations: paper beam a; PET beam with a mass on the tip $\mathbf{b}$. The curled stable equilibrium configurations represented here are discretized in $N_{e}=\{10,20,30,40\}$ elements.

and the moment of inertia of its section. Also, this approximation seems rather precise for our comparative purposes. Therefore, we will estimate the bending stiffness coefficient for the rotational spring in the Hencky model by assuming that in this spring there is the same deformation energy which one can find in the corresponding beam element, as calculated with the De Saint Venant solutions. However, we are aware of the fact that the a priori estimate of the Young modulus of used paper and used PET strips could be too approximate to be useful. Instead much more precise can be the $a$ priori estimate of the moment of inertia of considered strips. Therefore, we decided to get the Young moduli by means of the best fit of the equilibrium curled shapes as measured in our static experiments (see Fig. 5).

Indeed, to start with, we considered the local-minimum energy configurations which we called 'curled' for the two soft beams used in our experimentation (see Fig. 3). In the second series of figures we have overlapped to the image of the true 
physical object the calculated curve of equilibrium for the Hencky-type model (recall it is a Polygonal chain constituted by 30 segments). To be more precise: they show the photographic pictures of the curled equilibrium shapes of the physical stripes used in the experiment overlapped to the theoretical equilibrium configurations found by calculating the second lowest local minimum of the total energy $\Psi$ as evaluated with $N_{e}=30$.
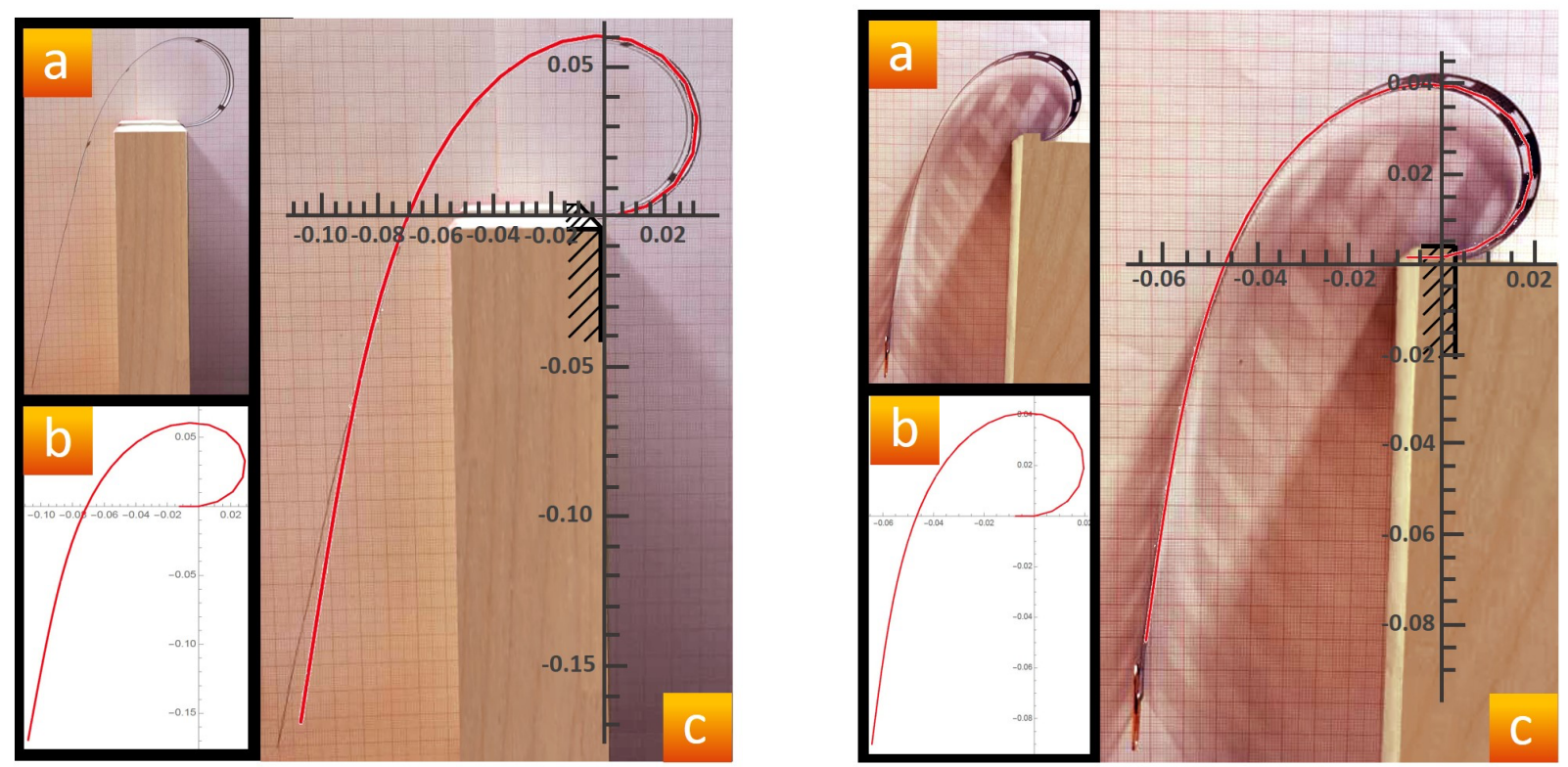

a)

Fig. 5 Experimentally observed and numerically evaluated equilibrium configurations: paper beam a; PET strip with a tip mass $\mathbf{b}$.

The agreement exhibited in Fig. 5, could be finally obtained by assuming for the lumped bending stiffness an expression which is a trivial consequence of the previously listed assumptions:

$$
Y_{b}=\kappa_{b i} \eta\left(j_{b}\right)^{-1}
$$

where $j_{b}$ is the second moment of area of the beam cross section. The obtained $Y_{b}$, as estimated by imposing the overlapping of the figures by varying in a 'reasonable' range the values for $\kappa_{b i}$, can be regarded as the best approximation of the the Young modulus of the considered materials, which we can obtain.

This value will be used now on, in this paper, as it identifies the most correct material parameter. Particularly, we found for the paper beam the best fitted value is given by $Y_{b}=1.25 \mathrm{GPa}$ while for the PET beam such value is given by $Y_{b}=2.5 \mathrm{GPa}$. Of course we started, in the guessing procedure, from tentative values in an interval including to the otherwise measured values of the Young moduli for paper and PET.

We remark here that the constitutive law (4) is just one of the possible choice to describe the behavior of the considered soft beam. As a matter of fact, many other constitutive laws can be adopted as, for instance, a quadratic function of the 'deformation' angle $\phi_{i}$ with the expression:

$$
\Psi_{e l}{ }^{\text {Quad }}=\sum_{i=1}^{N_{e}} \kappa_{b i} \frac{\phi_{i}{ }^{2}}{2}
$$

or also a trigonometric dependence on the same angle as follows:

$$
\Psi_{e l}{ }^{T r g}=\sum_{i=1}^{N_{e}} \kappa_{b i}\left[1-\cos \left(\phi_{i}\right)\right]
$$

Indeed, the law (12) is the most commonly adopted one, while the law (13) some times is preferable because it is easier for computing purpose when Cartesian coordinates are used as Lagrangian variables but in the validity range between $-\pi / 2$ and $\pi / 2$, values excluded. The choice of the proper constitutive law is quite difficult and deserves a careful procedure based on experimental observation. For instance, procedures which make use of the $\chi$-square test can be employed to select the proper law. It is also worthy to say that if the deformations are small, all the considered laws produce the same results 


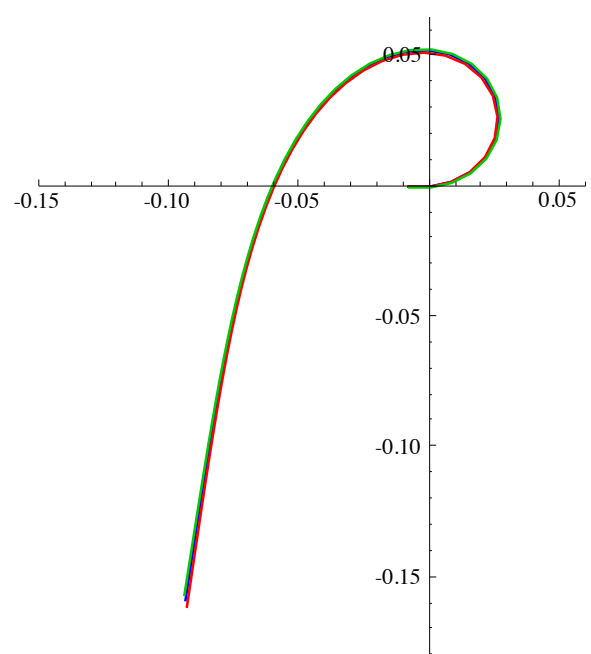

a)

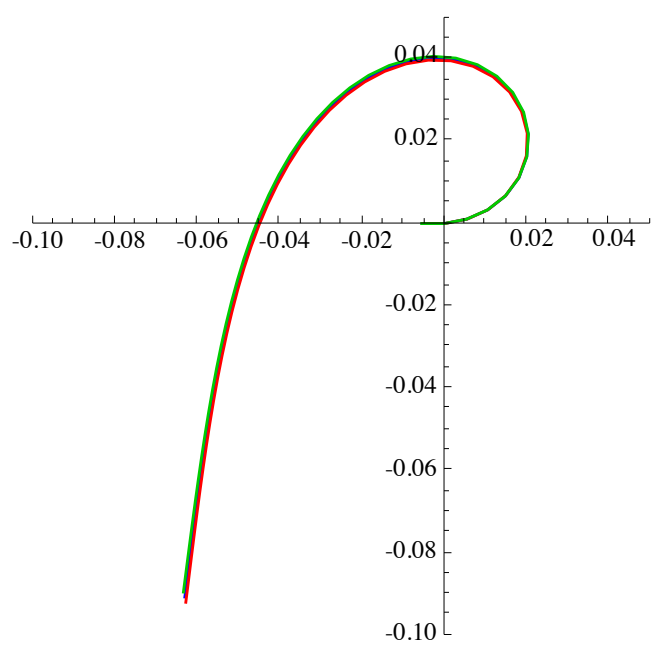

b)

Fig. 6 Curled stable equilibrium configurations with different constitutive laws: quadratic elastic potential (blue solid line); cosine elastic potential (red solid line); hyperbolic cosine elastic potential (green solid line). Paper beam a; PET beam with a tip mass b. The curled stable equilibrium configurations represented here are discretized in $N_{e}=40$ elements.

Table 1 Comparison between Hencky and Euler-Bernoulli model for the paper cantilever strip: natural frequencies.

\begin{tabular}{lcccc}
\hline & mode I & mode II & mode III & mode IV \\
\hline Hencky model $(\mathrm{Hz})$ & 0.3687 & 2.3121 & 6.4774 & 12.7002 \\
Euler-Bernoulli model $(\mathrm{Hz})$ & 0.3810 & 2.3877 & 6.6857 & 13.1014 \\
Relative error $(\%)$ & -3.23 & -3.17 & -3.12 & -3.06 \\
\hline
\end{tabular}

Table 2 Comparison between Hencky and Euler-Bernoulli model for the PET cantilever strip: natural frequencies.

\begin{tabular}{lcccc}
\hline & mode I & mode II & mode III & mode IV \\
\hline Hencky model (Hz) & 0.3953 & 3.2433 & 9.8290 & 19.9172 \\
Euler-Bernoulli model (Hz) & 0.4002 & 3.2644 & 9.9438 & 20.3689 \\
Relative error $(\%)$ & -1.22 & -0.65 & -1.15 & -2.22 \\
\hline
\end{tabular}

since they have the same main leading term in a Taylor expansion. In our case, we compared the curled stable equilibrium configurations obtained for the three mentioned laws (see Fig. 6) and thus, we can establish that even if the displacements and the rotations are large, the deformations, on the contrary, are almost in a small range. For this reason, in the present study we can adopt any law among those considered.

\subsection{Free linearized vibrations of the undamped soft beams}

For the sake of dynamic analysis, and to check the convergence of the discrete formulation proposed, we also perform a modal linearized analysis of the two examined cases. A first investigation has been performed comparing the results of the proposed Hencky-type formulation with those obtained by the standard Euler-Bernoulli model. By neglecting the gravitational potential and assuming a discretization of $N_{e}=30$ rigid elements, we can easily compare the natural frequencies of the cantilever beams for the two above-mentioned formulations in the undamped case. Tables 1 and 2 summarize these results, limited to the first four modes, for the paper strip and for the PET beam with a point mass attached to its tip, respectively. As it can be seen in these tables, the relative error between the continuous and the discrete model, with only 30 elements, at most is about $3 \%$, therefore we have a satisfactory accuracy even with relatively few elements.

Subsequently, the free vibrations of the considered soft beams have been analyzed around equilibrium configurations. In particular, in the selected cases we found that each beam under a uniform gravitational load presents two stable equilibrium configurations which are solutions of the following equation:

$$
\left.\frac{\partial \Psi}{\partial \Phi_{i}}\right|_{\overline{\mathbf{\Phi}}}=0 \quad \forall i=1, . . N_{e}
$$


Table 3 Natural frequencies of free vibrations around the global and local equilibrium shape in the paper strip case.

\begin{tabular}{lcccc}
\hline & mode I & mode II & mode III & mode IV \\
\hline Global equilibrium $(\mathrm{Hz})$ & 1.147 & 3.225 & 6.466 & 11.949 \\
Local equilibrium $(\mathrm{Hz})$ & 1.250 & 1.629 & 4.141 & 8.701 \\
\hline
\end{tabular}

Table 4 Natural frequencies of free vibrations around the global and local equilibrium shape in the PET strip case.

\begin{tabular}{lcccc}
\hline & mode I & mode II & mode III & mode IV \\
\hline Global equilibrium $(\mathrm{Hz})$ & 1.285 & 4.298 & 9.217 & 18.463 \\
Local equilibrium $(\mathrm{Hz})$ & 1.455 & 1.944 & 6.003 & 13.509 \\
\hline
\end{tabular}

The two stable solutions ( $\overline{\mathbf{\Phi}})$ of Eq. (14) are a global minimum and a local one: the former being the 'classical' solution of a cantilever beam which resembles a hanging wire, and the latter is the 'curled' shape shown in Fig. 3. To study the small vibrations around these equilibrium shapes, we linearize the equations of motion or in other words, we reduce the potential energy $\Psi$ and kinetic energy $\mathfrak{K}$ to quadratic forms considering the leading terms (of order two) in a Taylor expansion of these energies as computed below

$$
\begin{gathered}
\Psi^{*}=\left.\frac{1}{2} \frac{\partial^{2} \Psi}{\partial \Phi_{i} \partial \Phi_{j}}\right|_{\overline{\mathbf{\Phi}}} \Phi_{i} \Phi_{j} \\
\mathfrak{K}^{*}=\left.\frac{1}{2} \frac{\partial^{2} \mathfrak{K}}{\partial \dot{\Phi}_{i} \partial \dot{\Phi}_{j}}\right|_{\overline{\mathbf{\Phi}}} \dot{\Phi}_{i} \dot{\Phi}_{j}
\end{gathered}
$$

From the matrices associated to the quadratic forms (16) and (15), i.e.,

$$
M_{h k}=\left.\frac{\partial^{2} \mathfrak{K}}{\partial \dot{\Phi}_{h} \partial \dot{\Phi}_{k}}\right|_{\overline{\mathbf{\Phi}}} \quad \text { and } \quad U_{h k}=\left.\frac{\partial^{2} \Psi}{\partial \Phi_{h} \partial \Phi_{k}}\right|_{\overline{\mathbf{\Phi}}},
$$

we may compute the eigenvalues and the eigenvectors associated and report them in the tables 3 and 4 and in Figs. 7 and 8 , respectively, for both cases: the paper and the PET strip with a point mass on the tip. Specifically, we note that comparing the first natural frequencies of the global minimum and of the local minimum, the frequency of the local minimum solution is larger than the other one. Thus, in a sense, it is as if the vibrating length for the curled solution is smaller than the other one and for this reason, the beam behaves a bit more stiffly. We observe also that the natural modes, in Figs. 7 and 8 , exhibit a number of nodes, which are resting points, progressively growing with the number of modes as it is common in vibrating problems.

\subsection{Motions in the neighborhood of the curled stable equilibrium configurations: large oscillations}

To prove experimentally that the observed equilibrium curled configuration is indeed stable it is necessary to observe the small motions starting in its neighborhood and establish that they are not increasing definitively the actual configuration from the curled equilibrium. No observed small perturbation has caused such a definitive removal. The used numerical code is capable to solve any initial value problem by forecasting the evolution of any in-plane oscillating motion consequent to any initial displacement of the extreme tip of the strip. To make simple the experimental determination of the needed initial data, we have considered only motions with initial zero velocity.

From the experimental pictures of said initial configuration, we evaluate the equilibrium configuration corresponding to the imposed placement of the terminal strip tip. By minimizing the energy and by using the already determined constitutive parameters we can calculate the effective Lagrangian coordinates of the observed initial configurations.

In Fig. 9 we can observe the effective overlap of the computed piecewise linear curve predicting the shape of the strip and the real picture of the both the strips considered. One has to remark that by using always and only the few parameters determined with the first fitting, the one which allowed for the prediction of the curled shape, we have obtained always a very close correspondence between experimental measurements and theoretical predictions. In the second step of the procedure we have specified as initial values these calculated equilibria, together with a condition of vanishing of all initial Lagrangian velocities.

By solving the ODEs given by Eqs. (8) we can predict the motions which originate from the experimentally imposed initial conditions. In order to present the obtained computed motions, in terms of the time evolutions of the orientations 


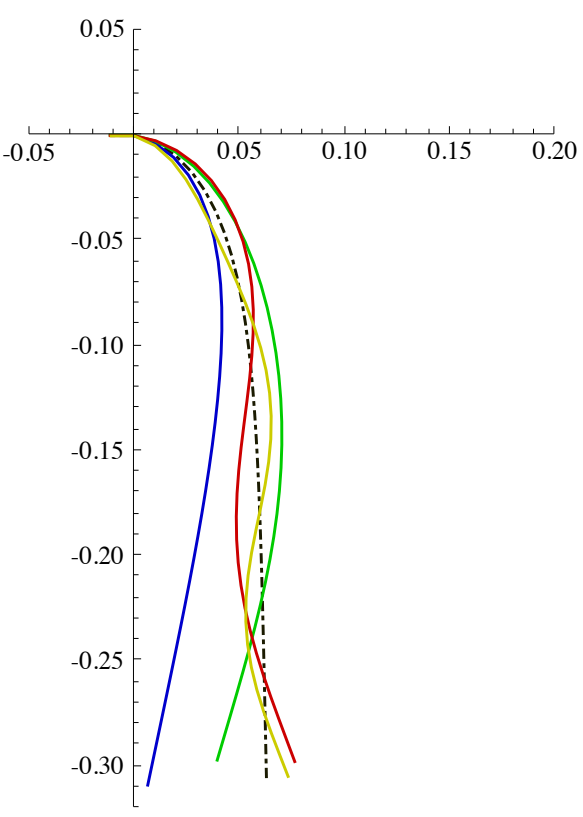

a)

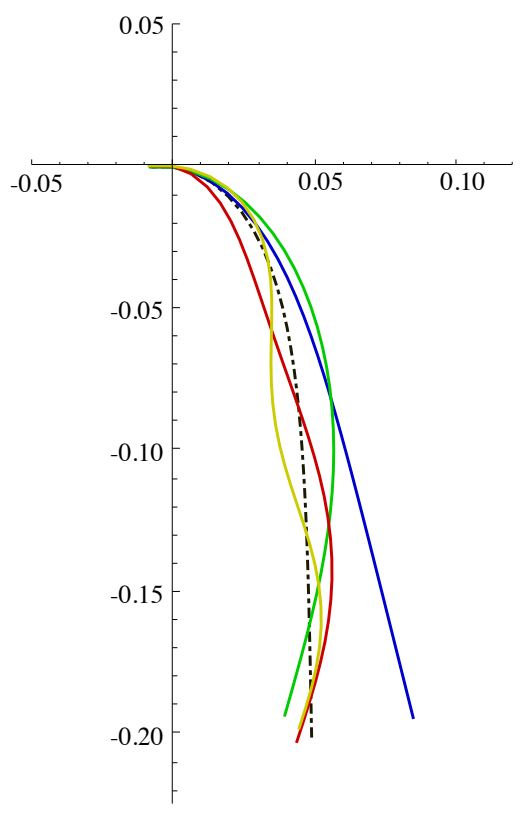

b)

Fig. 7 First four natural modes (solid lines) around the global equilibrium shape (dot-dashed line). Paper beam a; PET beam with a mass on the tip b. Legend: blue line corresponds to the first mode; green line to the second mode; red line to the third mode; yellow line to the fourth mode.
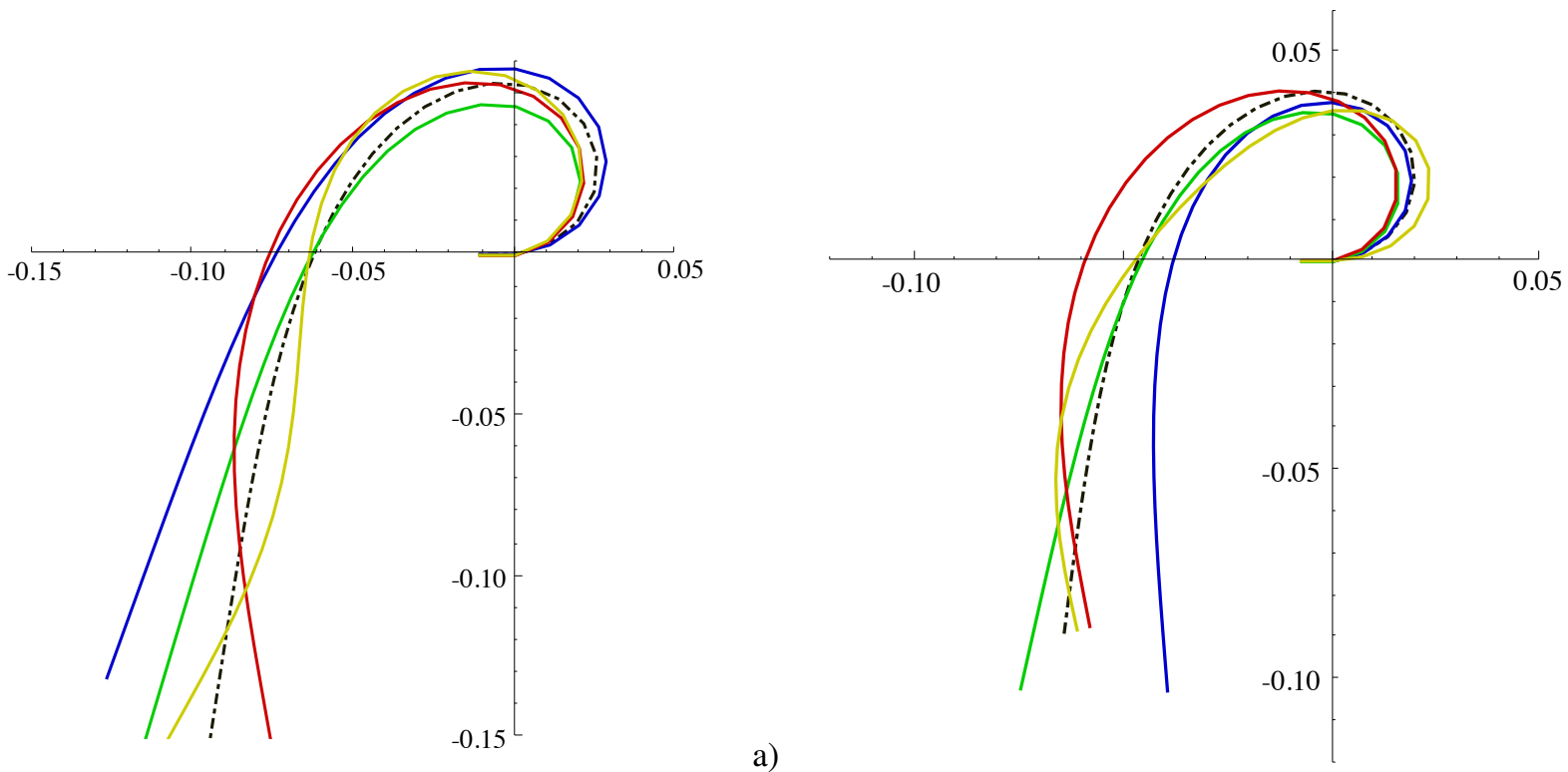

b)

Fig. 8 First four natural modes (solid lines) around the local 'curled' equilibrium shape (dot-dashed line). Paper beam a; PET beam with a mass on the tip b. Legend: blue line corresponds to the first mode; green line to the second mode; red line to the third mode; yellow line to the fourth mode.

$\Phi_{i}(t)$ and their derivatives velocities $\dot{\Phi}_{i}(t)$ we have elaborated the Fig. 10 and Fig. 11, respectively. They account for both the two cases studied here: that is the paper and the PET strip beams.

Figure 11 is then presented to show, by reducing the time interval, the initial part of the predicted motion.

There are some remarks which are made possible by the results of our numerical simulations. These remarks may be considered as some interesting conjectures which may conduct to some rigorous demonstrations.

1. The considered initial shapes and the calculated equilibrium configurations, towards which the system during its evolution, is approaching, are all curves whose curvature has a constant sign. This means that said curves are not crossing their tangents. This has as a consequence that the coordinates $\Phi_{i}(t)$, in every instant $t$, are not decreasing 
with the increasing index $i$. Indeed, we remark that Fig. 10 shows the calculated values of considered Lagrangian coordinates. The corresponding plots show how the sequence $\Phi_{1}(t)$ to $\Phi_{N e}(t)$ is not decreasing.

2. Always Fig. 10 shows that when the index $i$ is increasing, the values of $\Phi_{i}(t)$ tend to be constant. This means, geometrically, that the final part of the beam tends to remain straight.

3. In Fig. 11 we see the plots of Lagrangian (in this case angular) velocities. These velocities have a maximum value in the beam segments characterized by low values of the index $i$ : i.e. when the considered segments of the vibrating beams are close to the clamped one and when their characterizing abscissa $s$ is smaller than $L / 4$. The velocities are larger at the beginning of the calculated motions. Subsequently, the dissipation phenomena become dominant and the time evolution produces all vanishing Lagrangian velocities. This means that all plots of Lagrangian velocities are increasingly superimposed and crowded. A simple inspection of Fig. 11 allows us to establish which segment has the highest velocity at every time instant.

All presented numerical simulations incorporate a description of the dissipation phenomena which are occurring during the performed experiments. Said simulations were performed by using some estimated viscosity coefficients for the considered soft beams obtained. This estimate was obtained by means of a first, rather rough, but surprisingly efficient, criterion. We observed that the stopping time of the vibrating beams can be reasonably well detected by using the videos which were recorded during the experiments, even if there is, at the moment, a large sensitivity error. A simple best-fit determination of viscosity parameters has been performed to get a numerical estimation of the stopping times which are in a reasonable agreement with the measured times.

In particular we have found that

for the strip constituted by paper and

$$
c_{n}=2.0 \times 10^{-5} \mathrm{Nm} \mathrm{s}
$$

$$
c_{n}=7.0 \times 10^{-5} \mathrm{Nm} \mathrm{s}
$$

when the strip constituted by PET is considered.

Once this first estimate has been successfully obtained it was possible to produce the following Fig. 12. In it, one can find the plots describing the evolution, during the motion, of the total kinetic energy and the total potential energy for both the paper and the PET strips. To compare these calculated quantities with some experimentally measured physical quantity will require the development of more sophisticated experimental set-ups. However one can analyze them qualitatively. Their time evolution is exactly showing the expected features: i) they evolve in counter-phase, i.e. in time there is an oscillation of the beams in which kinetic energy is transformed into potential energy and vice versa ii) the kinetic energy decreases and finally tends to zero, as clearly had to happen because of dissipation iii) the potential energy approaches its value, as calculated for the local minimum which is estimated for the equilibrium configuration to which the beam is approaching after the needed number of damped oscillations.

To resume the whole calculated time history of Lagrangian coordinates we use the Fig. 13. This figure is conceived to show the trajectory in the so-called phase space of the Lagrangian coordinate of the terminal Hencky segment of considered beams, i.e. for both the paper strip and the PET strip. In other words, we present the phase space trajectory of the Lagrangian coordinate $\Phi_{N e}(t)$ for both the investigated cases. We do not find meaningful to show the similar trajectories relative to the other Lagrangian coordinates as they are very similar. We remark that: i) in the initial part of both the trajectories both motions are strongly affected by nonlinear phenomena ii) mentioned nonlinearities are more relevant for the paper strip when compared with the PET strip iii) towards the final part of the oscillations both systems become substantially linear and one can easily detect the configuration of stable equilibrium.

\section{Some concluding remarks and future challenges we expect to confront}

The motivations from which this paper had its origins are to be found in the study of fundamental constituents of the microstructure of pantographic metamaterials. It was surprising to discover that in the literature: i) problems of motion and deformation of beam elements under the effect of distributed loads (may these loads be uniform or not uniform) have not been extensively studied; ii) very few numerical and theoretical results are available; iii) to our knowledge no experimental evidence of some nonstandard (but stable) equilibrium configurations for said beams have been described. On the other hand, the dynamics of pantographic metamaterials (see [42,85]) seems to promise very interesting developments and therefore the dynamical phenomena occurring to their elementary constituents (i.e. beams with distributed loads) seems necessary. The main result of the present paper consists in showing experimentally that both the local minima for the total energy of Euler-Bernoulli inextensible highly-flexible beams which have been predicted in [36] correspond to real, effectively observed, equilibrium configurations for some physical objects (PET or paper strips). While the global minimum 

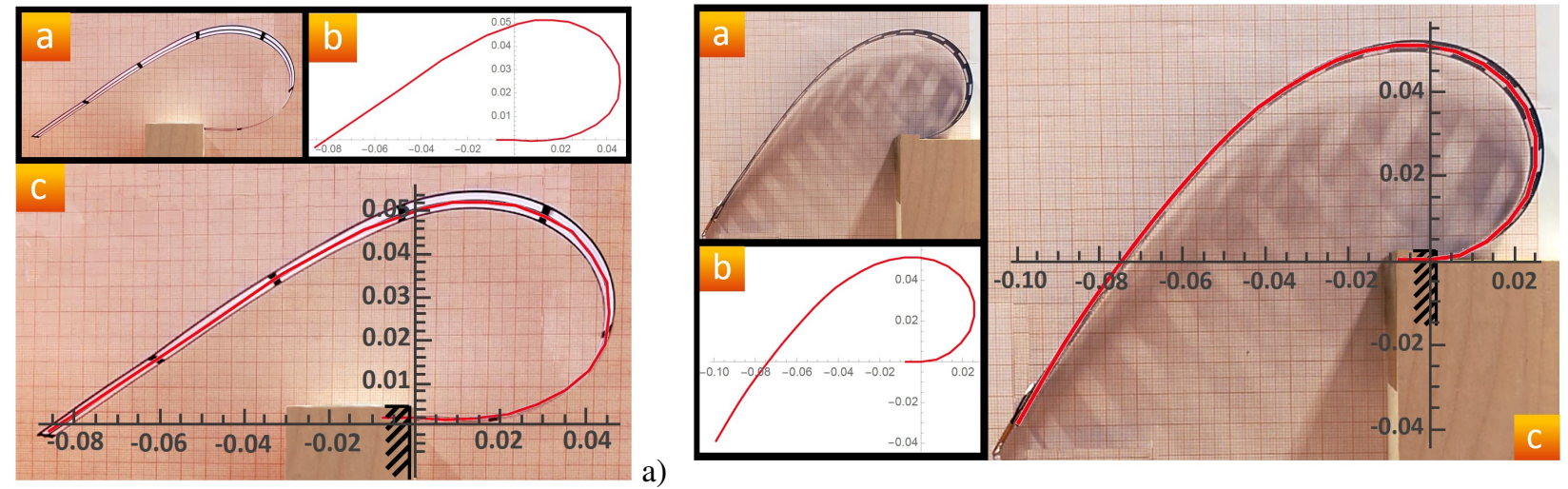

a)

Fig. 9 Experimentally imposed and numerically evaluated initial configurations: paper beam a; PET beam with a mass on the tip $\mathbf{b}$.
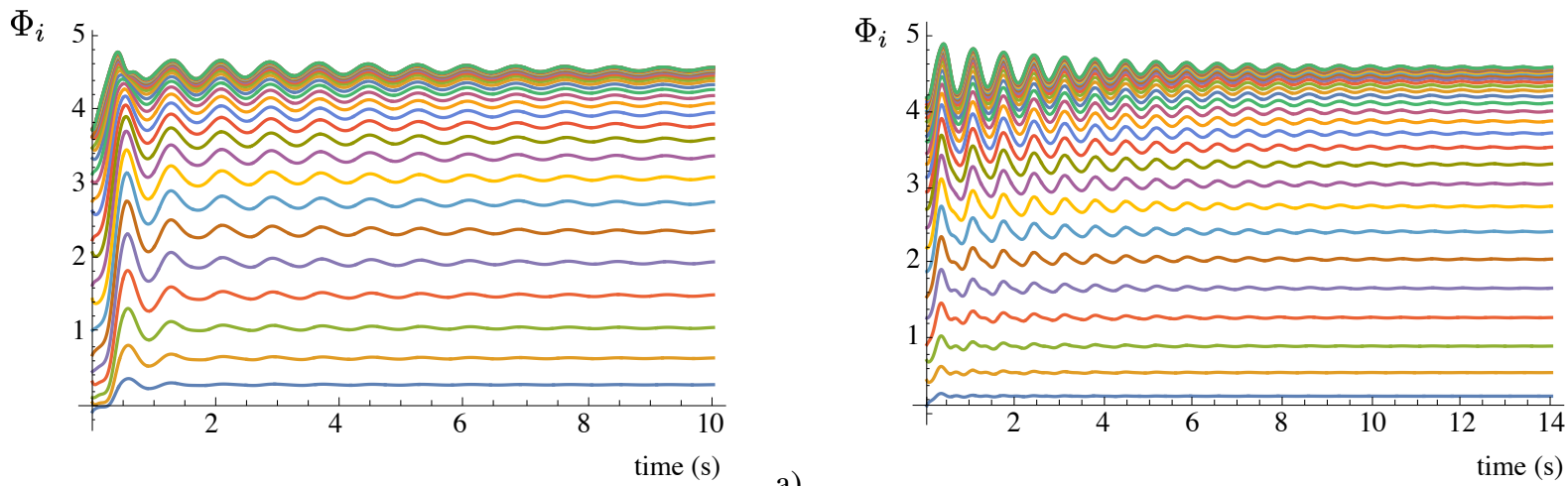

a)

Fig. 10 Time history of the Lagrangian coordinates, $\Phi_{i}(t)$, around the curled equilibrium configurations for the paper case a, and for the PET case b. Their plots result in an increasing order from the $\Phi_{1}(t)$ to $\Phi_{N e}(t)$.
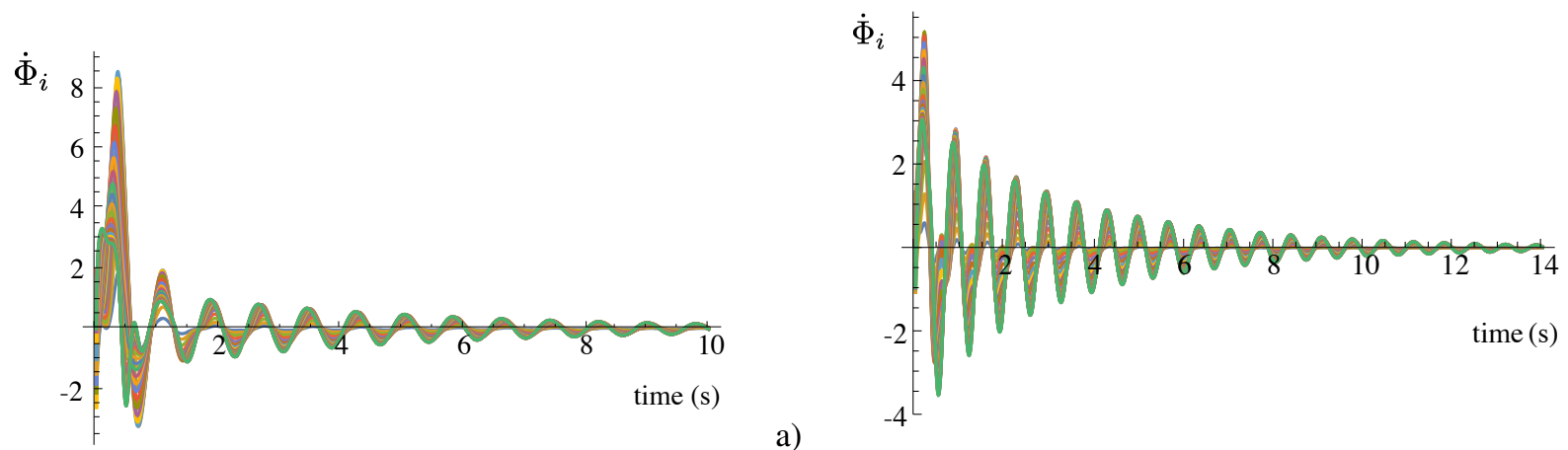

a)

b)

Fig. 11 Time history of the angular velocities, $\dot{\Phi}_{i}(t)$ for the paper case a, and for the PET case $\mathbf{b}$.

corresponds to a configuration which is expected and rather standard (we could call it the configuration 'like the pending strip', the other theoretically observed local minimum corresponds to a (nonstandard) curled shape. In the present paper, we show with a very simple experimental apparatus that the curled equilibrium shapes can be observed experimentally. Moreover, we show that, by means of a simple Lagrangian Hencky-type directly discrete model, it is rather easy to predict numerically both the observed equilibrium shapes and the damped oscillations (non-linear and linear) in the neighborhood of considered configurations. Remark that we wanted to use directly a Hencky-type discrete model for the prediction of the dynamic behavior of used strips as we wanted to avoid any discretization of a continuous Euler-type model. The validity of this choice is supported, also, by the very first study on the subject which one can find in [37]. In any case the adopted Lagrangian formulation i) is computationally very efficient and ii) can be interpreted from a physical point of view in a very clear way (see the previous figures describing Hencky type-models). Moreover, Lagrangian discrete models are producing very effective systems of ODEs which can be easily used to predict the motion of considered systems in the most 

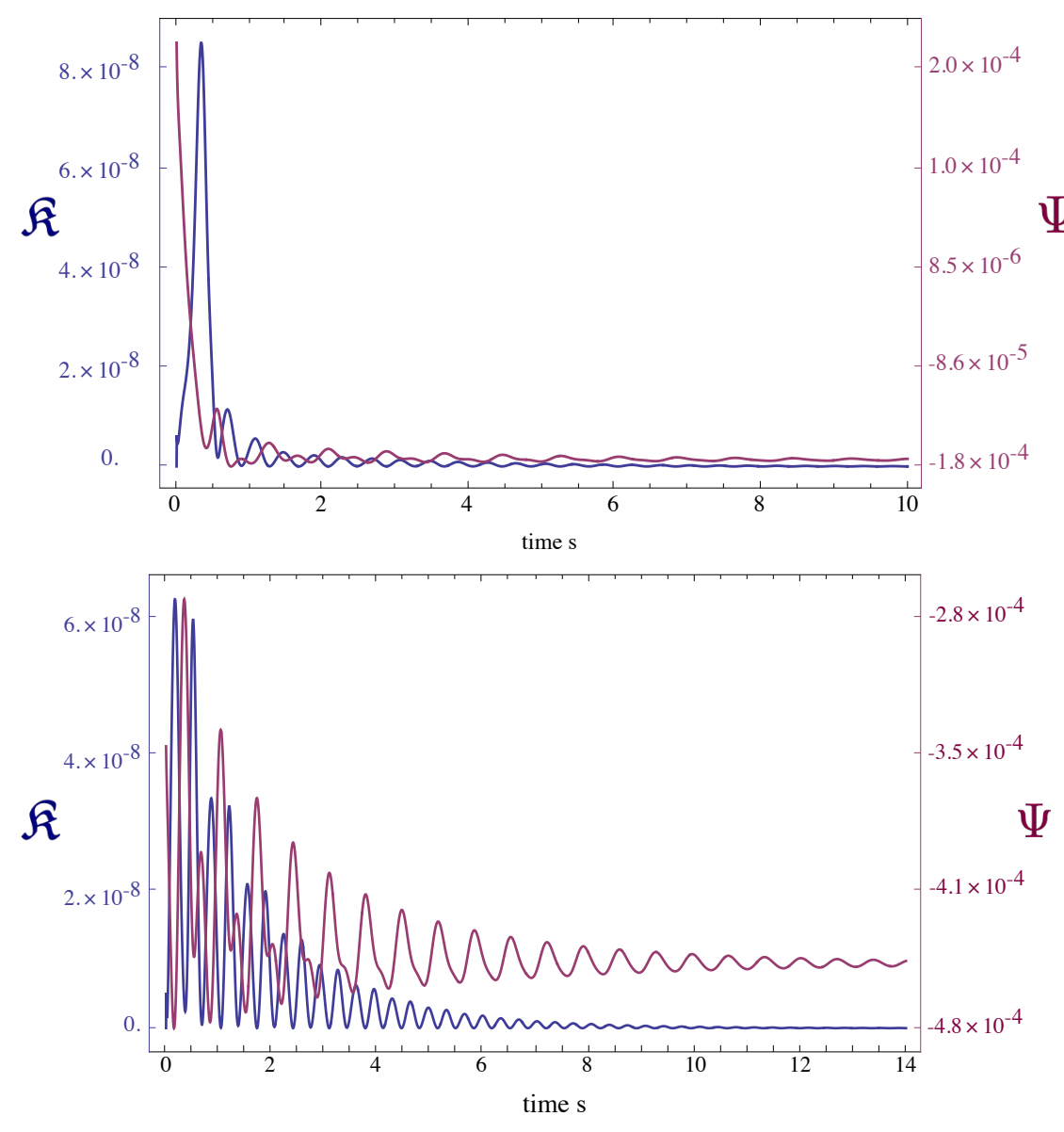

a)

b)

Fig. 12 Kinetic $\mathfrak{K}$ (blue solid line) and potential energy $\Psi$ (purple solid line) vs time graphs for the paper case a, and for the PET case $\mathbf{b}$.
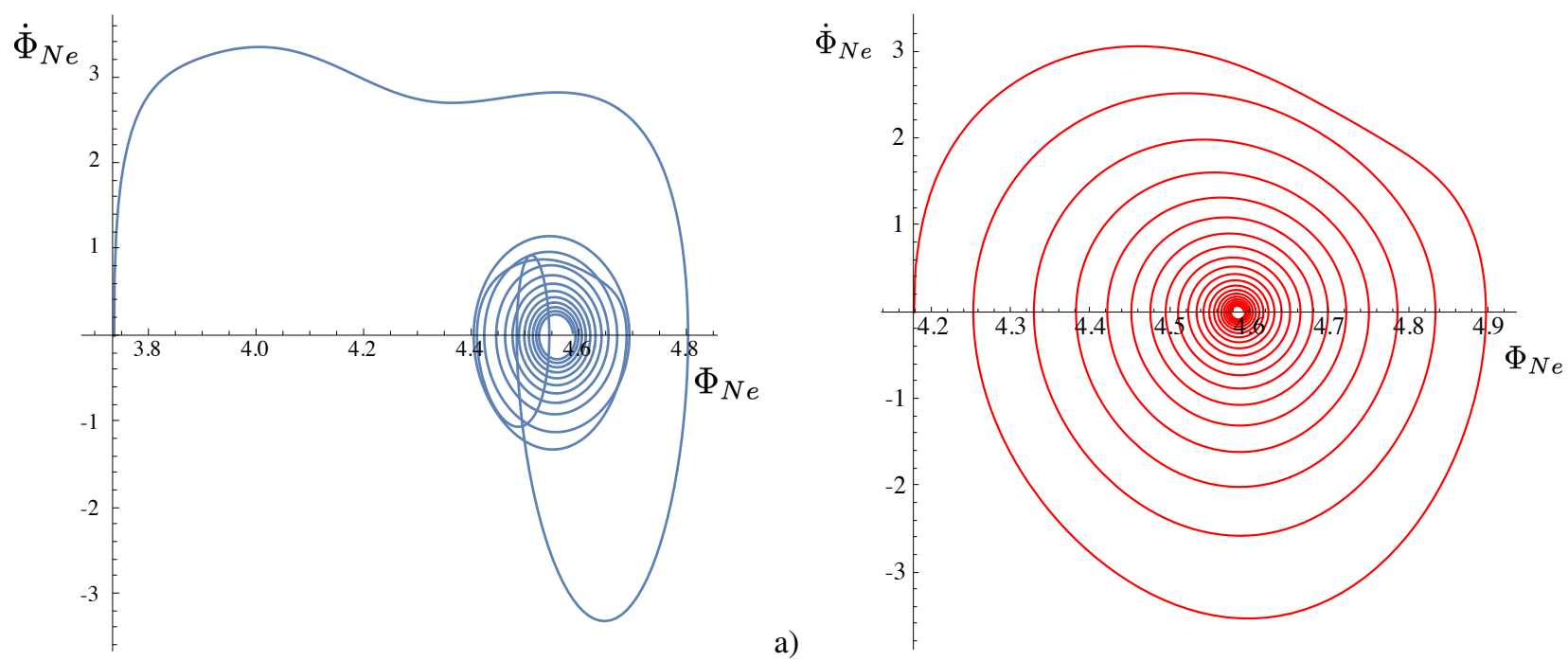

Fig. $13 \dot{\Phi}_{N e}$ vs $\Phi_{N e}$ phase paths of the paper case a, and of the PET case $\mathbf{b}$. 
general nonlinear regime. Besides, exactly by exploiting the said efficient features of Lagrangian discrete models, we could compare the numerically obtained solutions of the Lagrangian differential equations with the results of our experimental measurements. We have got a very good qualitative agreement (the reader can refer to the supplementary data which we have made available online: they include the videos of experimental observations and the animations obtained with our numerical codes. These videos are related to the examples in Figs. 10 and 11). However, for the limited set of measurements which we could get with the required precision, we also obtained a very close correspondence between the theoretical predictions and the observed physical quantities. The reader has to remark also that the quantitative and qualitative agreement which has been obtained is based on the identification of very few constitutive parameters and that the Hencky-type discrete model has to be compared with the discretization of Euler continuous beam obtained by introducing few finite elements or isogeometric schematizations involving few degrees of freedom.

It can be therefore concluded that even when more accurate experimental set-ups will be constructed and used the modeling procedure presented here has great chances to be carefully predictive.

The Hencky-type model is based on the choice of some lumped constitutive parameters, to be introduced in the postulated Lagrangian functions: we expect that even in presence of more sophisticated dynamic measurements these parameters can be efficiently fitted to get further precise and robust quantitative predictions (in this context see, e.g., [1, 88, 97]).

We are sure of the greater efficiency of Hencky-type models also when more complicated systems are considered (see [43]). Therefore we are confident that codes similar to the simple ones which were used in the present paper can be developed, and will be extremely useful, also in the dynamics study of i) Timoshenko beams [30,31,60], ii) lattice systems including many beam structural elements both in the case of 1D systems [7,67,90,113], in small or large deformations regimes [19], or in the case 2D and 3D structures [20,52,65,86,93,101,110], iii) more complex micro-structures which arise in the theory of metamaterials (see [53]). We believe that the alternative approach, based on the formulation of continuous models and on their subsequent discretization [18,23] although is substantially equivalent, may present some difficulties, when the discretization process of the continuous model is obtained without taking into account the physical nature of the modeled mechanical systems.

The theoretical analysis presented here allows for the determination of the sufficient criteria for which the curled equilibrium configuration can be experimentally observable (i.e. be stable). In this respect, one can note that the case of PET beam required a more sophisticated analysis. To get the stability of the curled configuration it was necessary to add a concentrated mass, i.e. the paper clip, at the free end of the strip. The distributed weight of PET strip was not enough and additional weight (we choose to place it at the free end) was required for making stable the 'curled equilibrium'. Another possibility would have been to use a longer strip, as 'by adding length' the nondimensional analysis of used Lagrange equations we could have achieved a stable equilibrium configuration also under its distributed own weight alone.

This analysis is confirmed by the observation that, after having removed the additional tip mass there is only one minimum of total energy and that the curled equilibrium configuration becomes unstable. In this case, there is not a second local minimum for total energy.

These results are confirmed by the analysis developed in [36] where it was shown that there exists a critical value for the applied external load under which only one minimum of the total energy exists.

This minimum is obviously related to the standard equilibrium configuration which is always observed (and often described in the literature) which is very similar to the 'pending wire shape' exhibited also by a flexible cantilever beam. To confirm further the aforementioned conclusion in Fig. 14 we show, for the constitutive parameters characterizing the experimentally used PET strip, that, in absence of the tip mass, the subsequent configurations as calculated in a stroboscopic motion. This motion is sampled at the rate of 10 images per second starting from an imposed initial configuration. This configuration is represented by a green solid line and is the same which was displayed in Fig. 9b. The minimum of the total energy represented the image of the current configuration captured at $1.4 \mathrm{~s}$ (red solid line). The interested reader will find a movie for this motion based on performed numerical simulation added as supplementary data in the website of the journal.

Acknowledgements LAI is supported by a grant from the Government of the Russian Federation (No. 14.Y26.31.0031). 


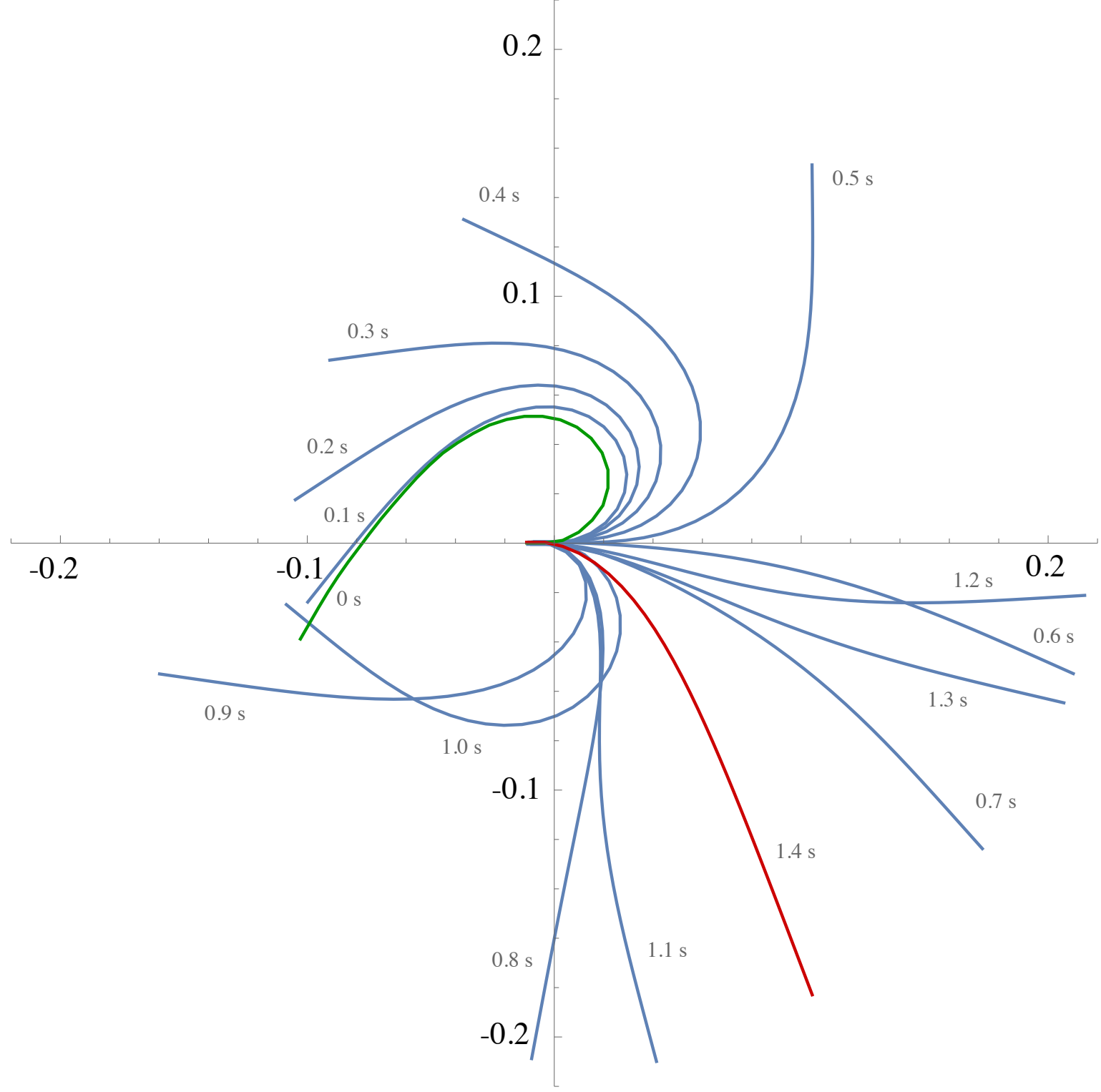

Fig. 14 Stroboscopic motion of the PET beam without mass on the tip. The initial shape is highlighted in green and the final one in red. The label near each configuration represents the attained time for the shown configuration.

\section{References}

[1] B.E. Abali, C.C. Wu, and W.H. Müller, An energy-based method to determine material constants in nonlinear rheology with applications, Continuum Mechanics and Thermodynamics 28(5), 1221-1246 (2016).

[2] H. Abdoul-Anziz and P. Seppecher, Homogenization of periodic graph-based elastic structures, Journal de l'École polytechnique-Mathématiques (2018).

[3] H. Abdoul-Anziz and P. Seppecher, Strain gradient and generalized continua obtained by homogenizing frame lattices, Mathematics and mechanics of complex systems 6(3), 213-250 (2018).

[4] J. J. Alibert, A. Della Corte, I. Giorgio, and A. Battista, Extensional elastica in large deformation as $\Gamma$-limit of a discrete 1D mechanical system, Zeitschrift für angewandte Mathematik und Physik 68(2), 19 pages (2017).

[5] J. J. Alibert, A. Della Corte, and P. Seppecher, Convergence of Hencky-type discrete beam model to Euler inextensible elastica in large deformation: rigorous proof, in: Mathematical Modelling in Solid Mechanics, (Springer, 2017), pp. 1-12.

[6] J. J. Alibert, P. Seppecher, and F. dell'Isola, Truss modular beams with deformation energy depending on higher displacement gradients, Mathematics and Mechanics of Solids 8(1), 51-73 (2003).

[7] H. Altenbach, M. Bîrsan, and V. A. Eremeyev, On a thermodynamic theory of rods with two temperature fields, Acta Mechanica 223(8), 1583-1596 (2012).

[8] H. Altenbach, M. Bîrsan, and V. A. Eremeyev, Cosserat-type rods, in: Generalized Continua from the Theory to Engineering Applications, (Springer, 2013), pp. 179-248. 
[9] H. Altenbach and V. A. Eremeyev, On the constitutive equations of viscoelastic micropolar plates and shells of differential type, Mathematics and Mechanics of Complex Systems 3(3), 273-283 (2015).

[10] U. Andreaus, P. Baragatti, and L. Placidi, Experimental and numerical investigations of the responses of a cantilever beam possibly contacting a deformable and dissipative obstacle under harmonic excitation, International Journal of Non-Linear Mechanics 80, 96-106 (2016).

[11] U. Andreaus and P. Casini, Identification of multiple open and fatigue cracks in beam-like structures using wavelets on deflection signals, Continuum Mechanics and Thermodynamics 28(1-2), 361-378 (2016).

[12] U. Andreaus, M. Spagnuolo, T. Lekszycki, and S. R. Eugster, A Ritz approach for the static analysis of planar pantographic structures modeled with nonlinear Euler-Bernoulli beams, Continuum Mechanics and Thermodynamics 30, 1103-1123 (2018).

[13] S. S. Antman, Kirchhoff's problem for nonlinearly elastic rods, Quarterly of applied mathematics 32(3), 221-240 (1974).

[14] V. I. Arnol'd, Mathematical methods of classical mechanics (Springer Science \& Business Media, 2013).

[15] N. Auffray, F. dell'Isola, V. A. Eremeyev, A. Madeo, and G. Rosi, Analytical continuum mechanics à la Hamilton-Piola least action principle for second gradient continua and capillary fluids, Mathematics and Mechanics of Solids 20(4), 375-417 (2015).

[16] G. Ballio, Elastic-plastic dynamic behavior of a beam-column model, Meccanica 3(3), 177-186 (1968).

[17] G. Ballio, V. Petrini, and C. Urbano, The effect of the loading process and imperfections on the load bearing capacity of beam columns, Meccanica 8(1), 56-67 (1973).

[18] V. Balobanov and J. Niiranen, Locking-free variational formulations and isogeometric analysis for the timoshenko beam models of strain gradient and classical elasticity, Computer Methods in Applied Mechanics and Engineering 339, 137-159 (2018).

[19] E. Barchiesi, F. dell'Isola, M. Laudato, L. Placidi, and P. Seppecher, A 1D Continuum Model for Beams with Pantographic Microstructure: Asymptotic Micro-Macro Identification and Numerical Results, Advances in Mechanics of Microstructured Media and Structures, Vol. 87 (Springer, Cham, 2018).

[20] E. Barchiesi, G. Ganzosch, C. Liebold, L. Placidi, R. Grygoruk, and W. H. Müller, Out-of-plane buckling of pantographic fabrics in displacement-controlled shear tests: experimental results and model validation, Continuum Mechanics and Thermodynamics pp. 1-13 (2018).

[21] E. Barchiesi, M. Spagnuolo, and L. Placidi, Mechanical metamaterials: a state of the art, Mathematics and Mechanics of Solids(doi: 10.1177/1081286517735695) (2018).

[22] J. Bauer, L. R. Meza, T. A. Schaedler, R. Schwaiger, X. Zheng, and L. Valdevit, Nanolattices: An emerging class of mechanical metamaterials, Advanced Materials 29(40), 1701850 (2017).

[23] L. Beirão Da Veiga, T. J. R. Hughes, J. Kiendl, C. Lovadina, J. Niiranen, A. Reali, and H. Speleers, A locking-free model for Reissner-Mindlin plates: Analysis and isogeometric implementation via NURBS and triangular NURPS, Mathematical Models and Methods in Applied Sciences 25(08), 1519-1551 (2015).

[24] A. Bensoussan, J.L. Lions, and G. Papanicolaou, Asymptotic analysis for periodic structures (American Mathematical Soc., 2011).

[25] D. Bernoulli, Letter from Daniel Bernoulli to Euler, http://eulerarchive.maa.org/correspondence/letters/OO0147.pdf., 20 October 1742 .

[26] M. Bîrsan, H. Altenbach, T. Sadowski, V. A. Eremeyev, and D. Pietras, Deformation analysis of functionally graded beams by the direct approach, Composites Part B: Engineering 43(3), 1315-1328 (2012).

[27] M. Born, Untersuchungen über die Stabilität der elastischen Linie in Ebene und Raum, unter verschiedenen Grenzbedingungen, PhD thesis, University of Gottingen, 1906.

[28] B. B. Boubaker, B. Haussy, and J. F. Ganghoffer, Discrete models of woven structures. Macroscopic approach, Composites Part B: Engineering 38(4), 498-505 (2007).

[29] A. Carcaterra, F. dell'Isola, R. Esposito, and M. Pulvirenti, Macroscopic description of microscopically strongly inhomogenous systems: A mathematical basis for the synthesis of higher gradients metamaterials, Archive for Rational Mechanics and Analysis 218(3), 1239-1262 (2015).

[30] A. Cazzani, F. Stochino, and E. Turco, An analytical assessment of finite element and isogeometric analyses of the whole spectrum of Timoshenko beams, ZAMM-Zeitschrift für Angewandte Mathematik und Mechanik 96(10), 1220-1244 (2016).

[31] A. Cazzani, F. Stochino, and E. Turco, On the whole spectrum of Timoshenko beams. Part I: a theoretical revisitation, Zeitschrift für angewandte Mathematik und Physik 67(2), 24 (2016).

[32] J. Chróścielewski, R. Schmidt, and V. A. Eremeyev, Nonlinear finite element modeling of vibration control of plane rod-type structural members with integrated piezoelectric patches, Continuum Mechanics and Thermodynamics pp. 1-42 (2018).

[33] M. Cuomo, Forms of the dissipation function for a class of viscoplastic models, Mathematics and Mechanics of Complex Systems 5(3), 217-237 (2017).

[34] M. Cuomo, F. dell'Isola, and L. Greco, Simplified analysis of a generalized bias test for fabrics with two families of inextensible fibres, Zeitschrift für angewandte Mathematik und Physik 67(3), 61 (2016).

[35] M. Cuomo, F. dell'Isola, L. Greco, and N. L. Rizzi, First versus second gradient energies for planar sheets with two families of inextensible fibres: investigation on deformation boundary layers, discontinuities and geometrical instabilities, Composites Part B: Engineering 115, 423-448 (2017).

[36] A. Della Corte, F. dell'Isola, R. Esposito, and M. Pulvirenti, Equilibria of a clamped Euler beam (Elastica) with distributed load: Large deformations, Mathematical Models and Methods in Applied Sciences 27(08), 1391-1421 (2017).

[37] F. dell'Isola, U. Andreaus, A. Cazzani, R. Esposito, L. Placidi, U. Perego, G. Maier, and P. Seppecher, The complete works of Gabrio Piola: Volume II Commented English Translation (Springer Publishing Company, Incorporated, 2019). 
[38] F. dell'Isola, U. Andreaus, and L. Placidi, At the origins and in the vanguard of peridynamics, non-local and higher-gradient continuum mechanics: An underestimated and still topical contribution of Gabrio Piola, Mathematics and Mechanics of Solids 20(8), 887-928 (2015).

[39] F. dell'Isola, S. Bucci, and A. Battista, Against the fragmentation of knowledge: The power of multidisciplinary research for the design of metamaterials, in: Advanced Methods of Continuum Mechanics for Materials and Structures, (Springer, 2016), pp. 523-545.

[40] F. dell'Isola, M. Cuomo, L. Greco, and A. Della Corte, Bias extension test for pantographic sheets: numerical simulations based on second gradient shear energies, Journal of Engineering Mathematics 103(1), 127-157 (2017).

[41] F. dell'Isola, A. Della Corte, and I. Giorgio, Higher-gradient continua: The legacy of Piola, Mindlin, Sedov and Toupin and some future research perspectives, Mathematics and Mechanics of Solids 22(4), 852-872 (2017).

[42] F. dell'Isola, I. Giorgio, and U. Andreaus, Elastic pantographic 2D lattices: a numerical analysis on the static response and wave propagation, Proceedings of the Estonian Academy of Sciences 64(3), 219-226 (2015).

[43] F. dell'Isola, I. Giorgio, M. Pawlikowski, and N. L. Rizzi, Large deformations of planar extensible beams and pantographic lattices: heuristic homogenization, experimental and numerical examples of equilibrium, Proceedings of the Royal Society A 472(2185), 23 pages (2016).

[44] F. dell'Isola, T. Lekszycki, M. Pawlikowski, R. Grygoruk, and L. Greco, Designing a light fabric metamaterial being highly macroscopically tough under directional extension: first experimental evidence, Zeitschrift für angewandte Mathematik und Physik 66(6), 3473-3498 (2015).

[45] F. dell'Isola, A. Madeo, and P. Seppecher, Cauchy tetrahedron argument applied to higher contact interactions, Archive for Rational Mechanics and Analysis 219(3), 1305-1341 (2016).

[46] F. dell'Isola, G. Maier, U. Perego, U. Andreaus, R. Esposito, and S. Forest, The complete works of Gabrio Piola: Volume I Commented English Translation (Springer Publishing Company, Incorporated, 2014).

[47] F. dell'Isola and P. Seppecher, "Hypertractions and hyperstresses convey the same mechanical information continuum mech. thermodyn.(2010) 22: 163-176" by prof. Podio Guidugli and prof. Vianello and some related papers on higher gradient theories, Continuum Mechanics and Thermodynamics 23(5), 473-478 (2011).

[48] F. dell'Isola, P. Seppecher, J. J. Alibert, T. Lekszycki et al., Pantographic metamaterials: an example of mathematically driven design and of its technological challenges, Continuum Mechanics and Thermodynamics https://doi.org/10.1007/s00161-0180689-8, 1-34 (2018).

[49] F. dell'Isola, P. Seppecher, and A. Della Corte, The postulations á la D'Alembert and á la Cauchy for higher gradient continuum theories are equivalent: a review of existing results, Proceedings of the Royal Society A: Mathematical, Physical and Engineering Sciences 471(2183), 20150415 (2015).

[50] F. dell'Isola, P. Seppecher, and A. Madeo, How contact interactions may depend on the shape of Cauchy cuts in Nth gradient continua: approach "à la D'Alembert”, Zeitschrift für angewandte Mathematik und Physik 63(6), 1119-1141 (2012).

[51] F. dell'Isola, P. Seppecher, M. Spagnuolo, E. Barchiesi et al., Advances in pantographic structures: Design, manufacturing, models, experiments and image analyses, Continuum Mechanics and Thermodynamics (2019).

[52] F. dell'Isola and D. Steigmann, A two-dimensional gradient-elasticity theory for woven fabrics, Journal of Elasticity 118(1), $113-125$ (2015).

[53] dell'Isola F. and D. J. Steigmann (eds.), Discrete and Continuum Models for Complex (Meta) Materials (Cambridge University Press, In press).

[54] F. Dos Reis and J.F. Ganghoffer, Construction of micropolar continua from the asymptotic homogenization of beam lattices, Computers \& Structures 112, 354-363 (2012).

[55] F. Dos Reis and J. F. Ganghoffer, Equivalent mechanical properties of auxetic lattices from discrete homogenization, Computational Materials Science 51(1), 314-321 (2012).

[56] F. Dos Reis and J. F. Ganghoffer, Equivalent mechanical properties of auxetic lattices from discrete homogenization, Computational Materials Science 51(1), 314-321 (2012).

[57] F. Dos Reis and J.F. Ganghoffer, Homogenized elastoplastic response of repetitive 2D lattice truss materials, Computational Materials Science 84, 145-155 (2014).

[58] W. J. Duncan, A critical examination of the representation of massive and elastic bodies by systems of rigid masses elastically connected, The Quarterly Journal of Mechanics and Applied Mathematics 5(1), 97-108 (1952).

[59] M. Dupac and D. B. Marghitu, Nonlinear dynamics of a flexible mechanism with impact, Journal of sound and vibration 289(45), 952-966 (2006).

[60] I. Elishakoff, F. Hache, and N. Challamel, Variational derivation of governing differential equations for truncated version of bresse-timoshenko beams, Journal of Sound and Vibration 435, 409-430 (2018).

[61] V. A. Eremeyev, F. dell'Isola, C. Boutin, and D. Steigmann, Linear pantographic sheets: existence and uniqueness of weak solutions, Journal of Elasticity pp. 1-22 (2017).

[62] S. R. Eugster, C. Hesch, P. Betsch, and C. Glocker, Director-based beam finite elements relying on the geometrically exact beam theory formulated in skew coordinates, International Journal for Numerical Methods in Engineering 97(2), 111-129 (2014).

[63] L. Euler, De curvis elasticis, Additamentum I to his Methodus inveniendi lineas curvas maximi minimive proprietate gaudentes (Lausanne and Geneva, 1744).

[64] M. Ferretti, F. D'Annibale, and A. Luongo, Flexural-torsional flutter and buckling of braced foil beams under a follower force, Mathematical Problems in Engineering 2017 (2017).

[65] P. Franciosi, M. Spagnuolo, and O. U. Salman, Mean Green operators of deformable fiber networks embedded in a compliant matrix and property estimates, Continuum Mechanics and Thermodynamics pp. 1-32 (2018). 
[66] K. Frischmuth, W. Kosiéski, and T. Lekszycki, Free vibrations of finite-memory material beams, International Journal of Engineering Science 31(3), 385-395 (1993).

[67] S. N. Gavrilov, V. A. Eremeyev, G. Piccardo, and A. Luongo, A revisitation of the paradox of discontinuous trajectory for a mass particle moving on a taut string, Nonlinear Dynamics 86(4), 2245-2260 (2016).

[68] I. Giorgio and D. Del Vescovo, Non-linear lumped-parameter modeling of planar multi-link manipulators with highly flexible arms, Robotics 7(4), 60 (2018).

[69] I. Giorgio, N. L. Rizzi, and E. Turco, Continuum modelling of pantographic sheets for out-of-plane bifurcation and vibrational analysis, Proc. R. Soc. A 473(2207), 20170636 (2017).

[70] H. Goldstein, C. Poole, and J. Safko, Classical mechanics, 2002.

[71] L. Greco and M. Cuomo, Consistent tangent operator for an exact Kirchhoff rod model, Continuum Mechanics and Thermodynamics 27(4-5), 861-877 (2015).

[72] H. Hencky, Über die angenäherte lösung von stabilitätsproblemen im raum mittels der elastischen gelenkkette, Der Eisenbau 11, 437-452 (1920).

[73] A. Javili, Variational formulation of generalized interfaces for finite deformation elasticity, Mathematics and Mechanics of Solids 23(9), 1303-1322 (2018).

[74] A. Javili and A. D. Bakiler, A displacement-based approach to geometric instabilities of a film on a substrate, Mathematics and Mechanics of Solids https://doi.org/10.1177/1081286519826370 (2019).

[75] A. Javili, F. dell'Isola, and P. Steinmann, Geometrically nonlinear higher-gradient elasticity with energetic boundaries, Journal of the Mechanics and Physics of Solids 61(12), 2381-2401 (2013).

[76] A. Javili, B. Dortdivanlioglu, E. Kuhl, and C. Linder, Computational aspects of growth-induced instabilities through eigenvalue analysis, Computational Mechanics 56(3), 405-420 (2015).

[77] A. Javili, A. McBride, and P. Steinmann, Thermomechanics of solids with lower-dimensional energetics: on the importance of surface, interface, and curve structures at the nanoscale. a unifying review, Applied Mechanics Reviews 65(1), 010802 (2013).

[78] A. Javili, A. McBride, P. Steinmann, and B. Reddy, A unified computational framework for bulk and surface elasticity theory: a curvilinear-coordinate-based finite element methodology, Computational Mechanics 54(3), 745-762 (2014).

[79] S. Khakalo, V. Balobanov, and J. Niiranen, Modelling size-dependent bending, buckling and vibrations of $2 \mathrm{~d}$ triangular lattices by strain gradient elasticity models: applications to sandwich beams and auxetics, International Journal of Engineering Science 127, 33-52 (2018).

[80] S. Khakalo and J. Niiranen, Form II of Mindlin's second strain gradient theory of elasticity with a simplification: For materials and structures from nano-to macro-scales, European Journal of Mechanics-A/Solids 71, 292-319 (2018).

[81] F. A. Leckie and G. M. Lindberg, The effect of lumped parameters on beam frequencies, The Aeronautical Quarterly 14(3), 224-240 (1963).

[82] T. Lekszycki and Z. Mroz, On optimal support reaction in viscoelastic vibrating structures, Journal of Structural Mechanics 11(1), 67-79 (1983)

[83] A. Luongo, On the use of the multiple scale method in solving 'difficult' bifurcation problems, Mathematics and Mechanics of Solids 22(5), 988-1004 (2017).

[84] A. Luongo and F. D'Annibale, Nonlinear hysteretic damping effects on the post-critical behaviour of the visco-elastic beck's beam, Mathematics and Mechanics of Solids 22(6), 1347-1365 (2017).

[85] A. Madeo, A. Della Corte, L. Greco, and P. Neff, Wave propagation in pantographic 2D lattices with internal discontinuities, Proceedings of the Estonian Academy of Sciences 64(3S), 325-330 (2015).

[86] L. R. Meza, A. J. Zelhofer, N. Clarke, A. J. Mateos, D. M. Kochmann, and J. R. Greer, Resilient 3D hierarchical architected metamaterials, Proceedings of the National Academy of Sciences 112(37), 11502-11507 (2015).

[87] G. W. Milton, M. Briane, and D. Harutyunyan, On the possible effective elasticity tensors of 2-dimensional and 3-dimensional printed materials, Mathematics and Mechanics of Complex Systems 5(1), 41-94 (2017).

[88] A. Misra and P. Poorsolhjouy, Identification of higher-order elastic constants for grain assemblies based upon granular micromechanics, Mathematics and Mechanics of Complex Systems 3(3), 285-308 (2015).

[89] M. Nase, M. Rennert, K. Naumenko, and V. A. Eremeyev, Identifying traction-separation behavior of self-adhesive polymeric films from in situ digital images under t-peeling, Journal of the Mechanics and Physics of Solids 91, 40-55 (2016).

[90] J. Niiranen, V. Balobanov, J. Kiendl, and S. B. Hosseini, Variational formulations, model comparisons and numerical methods for Euler-Bernoulli micro-and nano-beam models, Mathematics and Mechanics of Solids 24(1), 312-335 (2019).

[91] C. Pideri and P. Seppecher, A homogenization result for elastic material reinforced periodically with high rigidity elastic fibres, Comptes Rendus de l'Academie des Sciences Series IIB Mechanics Physics Chemistry Astronomy 8(324), 475-481 (1997).

[92] C. Pideri and P. Seppecher, A second gradient material resulting from the homogenization of an heterogeneous linear elastic medium, Continuum Mechanics and Thermodynamics 9(5), 241-257 (1997).

[93] L. Placidi, E. Barchiesi, E. Turco, and N. L. Rizzi, A review on 2D models for the description of pantographic fabrics, Zeitschrift für angewandte Mathematik und Physik 67(5), 121 (2016).

[94] E. Reissner, On one-dimensional finite-strain beam theory: the plane problem, Zeitschrift für angewandte Mathematik und Physik ZAMP 23(5), 795-804 (1972).

[95] N. L. Rizzi, V. Varano, and S. Gabriele, Initial postbuckling behavior of thin-walled frames under mode interaction, Thin-Walled Structures 68, 124-134 (2013).

[96] G. Rosi and N. Auffray, Anisotropic and dispersive wave propagation within strain-gradient framework, Wave Motion 63, 120134 (2016). 
[97] G. Rosi, L. Placidi, and N. Auffray, On the validity range of strain-gradient elasticity: a mixed static-dynamic identification procedure, European Journal of Mechanics-A/Solids 69, 179-191 (2018).

[98] D. Rubinstein, Dynamics of a flexible beam and a system of rigid rods, with fully inverse (one-sided) boundary conditions, Computer methods in applied mechanics and engineering 175(1-2), 87-97 (1999).

[99] E. Ruocco, H. Zhang, and C. M. Wang, Hencky bar-chain model for buckling analysis of non-uniform columns, Structures 6, 73-84 (2016).

[100] S. Šalinić, An improved variant of Hencky bar-chain model for buckling and bending vibration of beams with end masses and springs, Mechanical Systems and Signal Processing 90, 30-43 (2017).

[101] D. Scerrato, I. A. Zhurba Eremeeva, T. Lekszycki, and N. L. Rizzi, On the effect of shear stiffness on the plane deformation of linear second gradient pantographic sheets, ZAMM - Zeitschrift für Angewandte Mathematik und Mechanik 96(11), 1268-1279 (2016).

[102] M. Spagnuolo and U. Andreaus, A targeted review on large deformations of planar elastic beams: extensibility, distributed loads, buckling and post-buckling, Mathematics and Mechanics of Solids(doi: 10.1177/1081286517737000) (2018).

[103] M. Spagnuolo, K. Barcz, A. Pfaff, F. dell'Isola, and P. Franciosi, Qualitative pivot damage analysis in aluminum printed pantographic sheets: numerics and experiments, Mechanics Research Communications 83, 47-52 (2017).

[104] D. J. Steigmann and F. dell'Isola, Mechanical response of fabric sheets to three-dimensional bending, twisting, and stretching, Acta Mechanica Sinica 31(3), 373-382 (2015).

[105] D. J. Steigmann and M. G. Faulkner, Variational theory for spatial rods, Journal of Elasticity 33(1), 1-26 (1993).

[106] E. Turco, Discrete is it enough? The revival of Piola-Hencky keynotes to analyze three-dimensional Elastica, Continuum Mechanics and Thermodynamics(doi: 10.1007/s00161-018-0656-4:1-19) (2018).

[107] E. Turco, F. dell'Isola, A. Cazzani, and N. L. Rizzi, Hencky-type discrete model for pantographic structures: numerical comparison with second gradient continuum models, Zeitschrift für angewandte Mathematik und Physik 67(4), 28 pages (2016).

[108] E. Turco, F. dell'Isola, N. L. Rizzi, R. Grygoruk, W. H. Müller, and C. Liebold, Fiber rupture in sheared planar pantographic sheets: Numerical and experimental evidence, Mechanics Research Communications 76, 86-90 (2016).

[109] E. Turco, I. Giorgio, A. Misra, and F. dell'Isola, King post truss as a motif for internal structure of (meta) material with controlled elastic properties, Royal Society open science 4(10), 20 pages (2017).

[110] E. Turco, M. Golaszewski, A. Cazzani, and N.L. Rizzi, Large deformations induced in planar pantographic sheets by loads applied on fibers: experimental validation of a discrete lagrangian model, Mechanics Research Communications 76, 51-56 (2016).

[111] C. M. Wang, H. Zhang, R. P. Gao, W. H. Duan, and N. Challamel, Hencky bar-chain model for buckling and vibration of beams with elastic end restraints, International Journal of Structural Stability and Dynamics 15(07), 16 pages (2015).

[112] Y. Wang and R. L. Huston, A lumped parameter method in the nonlinear analysis of flexible multibody systems, Computers \& structures 50(3), 421-432 (1994).

[113] S. T. Yaghoubi, V. Balobanov, S. M. Mousavi, and J. Niiranen, Variational formulations and isogeometric analysis for the dynamics of anisotropic gradient-elastic Euler-Bernoulli and shear-deformable beams, European Journal of Mechanics-A/Solids 69, 113-123 (2018).

[114] H. Zhang, C. M. Wang, and N. Challamel, Buckling and vibration of Hencky bar-chain with internal elastic springs, International Journal of Mechanical Sciences 119, 383-395 (2016). 